\title{
A Polarimetric Coherence Method to Determine Ice Crystal Orientation Fabric From Radar Sounding: Application to the NEEM Ice Core Region
}

Jordan, T. M.; Schroeder, Dustin M. ; Castelletti, D.; Li, J.; Dall, J.

Published in:

IEEE Transactions on Geoscience and Remote Sensing

Link to article, DOI:

10.1109/TGRS.2019.2921980

Publication date:

2019

Document Version

Peer reviewed version

Link back to DTU Orbit

Citation (APA):

Jordan, T. M., Schroeder, D. M., Castelletti, D., Li, J., \& Dall, J. (2019). A Polarimetric Coherence Method to Determine Ice Crystal Orientation Fabric From Radar Sounding: Application to the NEEM Ice Core Region. IEEE Transactions on Geoscience and Remote Sensing, 57(11), 8641-8657. https://doi.org/10.1109/TGRS.2019.2921980

\section{General rights}

Copyright and moral rights for the publications made accessible in the public portal are retained by the authors and/or other copyright owners and it is a condition of accessing publications that users recognise and abide by the legal requirements associated with these rights.

- Users may download and print one copy of any publication from the public portal for the purpose of private study or research.

- You may not further distribute the material or use it for any profit-making activity or commercial gain

- You may freely distribute the URL identifying the publication in the public portal 


\title{
A Polarimetric Coherence Method to Determine Ice Crystal Orientation Fabric from Radar Sounding: Application to the NEEM Ice Core Region
}

\author{
Thomas M. Jordan ${ }^{1,2}$, Dustin M. Schroeder ${ }^{1,3}$, Davide Castelletti ${ }^{1}, \mathrm{Jilu} \mathrm{Li}^{4}$, Jørgen Dall ${ }^{5}$.
}

\begin{abstract}
Ice crystal orientation fabric (COF) records information about past ice-sheet deformation and influences the present-day flow of ice. Polarimetric radar sounding provides a means to infer anisotropic COF patterns due to the associated birefringence of polar ice. Here we develop a polarimetric coherence (phase-based) method to determine horizontal properties of the COF. The method utilizes the azimuth and depth-dependence of the vertical gradient of the $h h v v$ coherence phase to infer the dielectric principal axes and birefringence which are then related to the second order fabric orientation tensor. Specifically, under the assumption that one of the orientational eigenvectors is vertical, we can determine the horizontal eigenvectors and the difference between the horizontal eigenvalues (a measure of horizontal fabric asymmetry). The method exploits single-polarized data acquired with varying antenna orientation. It applies to ground-based 'multi-polarization' surveys and is demonstrated using data acquired by CReSIS (Center for Remote Sensing of Ice Sheets) using MCRDs (Multi Channel Coherent Radar Depth Sounder) from the NEEM ice core region in Greenland. The analysis is validated using a combination of polarimetric matrix backscatter simulations and comparison with COF data from the NEEM ice core. The results are consistent with a conventional model of ice deformation at an ice divide where a lateral tension component is present, with minor horizontal COF asymmetry and the greatest horizontal concentration of crystallographic axes orientated near-parallel to the ice divide.
\end{abstract}

Index Terms-Radar Sounding, Polarimetry, Birefringence, Crystal Orientation Fabric (COF), Anisotropy, Ice cores, Icesheet dynamics.

\section{INTRODUCTION}

The net alignment of ice crystals in the polar ice sheets is referred to as the crystal orientation fabric (COF). Anisotropy in the COF provides a record of past ice deformation (strain history) [1]-[3] and vertical changes in the COF are often correlated with paleoclimate transitions [4], [5]. The viscosity of ice is dependent upon the orientation of the crystallographic axis ( $c$ axis) [6] and anisotropy in the COF has a pronounced effect upon present-day ice flow [7], [8]. Ground-truth knowledge of the COF exists only at a limited number of ice core sites which tend to be located at ice divides (e.g. [5], [9]). Additionally, due to rotation of ice sections in the drilling processes, ice cores cannot directly reveal fabric orientation in the horizontal plane [9], [10]. There is therefore a knowledge

\footnotetext{
1 Department of Geophysics, Stanford University, USA. ${ }^{2}$ School of Geographical Sciences, University of Bristol, UK. ${ }^{3}$ Department of Electrical Engineering, Stanford University, USA. ${ }^{4}$ Center for Remote Sensing of Ice Sheets, University of Kansas, USA. ${ }^{5}$ National Space Institute, Technical University of Denmark, Denmark.
}

gap regarding how the COF varies spatially across the polar ice-sheets.

It has been known since the 1970s that dielectric anisotropy associated with a horizontally asymmetric COF can be detected using polarimetric radar sounding [11], [12] and is manifest as two different electromagnetic phenomena. First; birefringent propagation (associated with horizontal anisotropy to the COF that varies smoothly with ice depth), and second; anisotropic scattering (associated with sharp depth transitions in the COF) [11], [13]. Dielectric anisotropy can be mathematically related to COF anisotropy via a normalized eigenvalue framework which provides a mapping between second order dielectric and orientation tensors [13]. Therefore, the eigenvectors and eigenvalues of the orientation tensor related to the dielectric principal axes and principal permittivities/birefringence - represent the central COF target variables from radar sounding.

Polarimetric power anomaly (also called depolarization) methods have conventionally been used to infer COF properties from ground-based radar sounding (e.g. [2], [11], [13][15]). Specifically, birefringent propagation results in azimuthal and phase/depth periodicity of the returned power as the measurement polarization plane is rotated, and these symmetry properties are used to infer COF properties. However, birefringent propagation results in 90 degree azimuthal power periodicity which means that inference of the dielectric principal axes/orientational eigenvectors is ambiguous (i.e. the azimuthal dependence is identical for a polarization plane aligned with either the lower or higher principal permittivity). Additionally, the presence of anisotropic scattering acts to break the 90 degree azimuthal symmetry, further complicating the analysis [2], [13]. Subsequently, it has been proposed that polarimetric phase-sensitive radar, provides a viable tool to unambiguously determine $\mathrm{COF}$ properties that relate to birefringence (i.e. smoothly varying horizontal anisotropy to the COF) [2], [16].

When there is a distribution of scatterers present, satellite applications of Polarimetric SAR (Synthetic Aperture Radar) often exploit coherence methods (e.g. [17], [18]). Rather than analyzing power, this approach involves statistically quantifying the phase correlation between orthogonal polarizations in a resolution cell and then using this as a basis to extract information. The application of a polarimetric coherence method to radar sounding in the context of COF determination was proposed by [19], [20] using quad-polarized (fully polarimetric) measurements from the airborne POLARIS (POLarimetric 
Airborne Radar Ice Sounder) radar system [21], [22]. In particular, [20] demonstrated that the hhvv coherence phase, (a statistical estimate of the relative phase between orthogonal copolarized measurements that have polarization planes parallel to the ice surface), exhibited a phase gradient with respect to ice depth that could be attributed to birefringent propagation. Subsequently, using data from the NEEM (North Greenland Eemian Ice Drilling) ice core region in Greenland, [20] used the vertical $h h v v$ phase gradient to estimate the horizontal asymmetry of the COF.

In this study we build upon the polarimetric coherence $\mathrm{COF}$ framework introduced by [19], [20] and adapt the technique for ground-based multi-polarization plane measurements (copolarized data as a function of azimuthal angle). This investigation is motivated by the general lack of quad-polarized radar sounding data, and the requirement to relate the coherence method to both a commonly used ground-based experimental set-up [2], [3], [13], [15], [16], [23] and the polarimetric backscatter models that have previously been used to interpret the ground-based data [2], [3], [13], [16].

We demonstrate the coherence method using data from the MCRDs (Multi Channel Coherent Radar Depth Sounder) radar system from three 'turning circles' in NEEM ice core region of Greenland. The full data set is described in detail by [14] who performed analysis of co-polarized and cross-polarized power. In this study we focus purely upon co-polarized data and show, for the case of multi-polarization plane measurements, that the coherence method is sufficient to determine horizontal fabric properties. Focusing purely on co-polarized data is advantageous as it represents the simplest ground-based experimental set-up, and therefore enables polarimetric surveys to be carried out faster and with a wider range of radar systems.

A central and novel feature of our investigation is the development of a polarimetric backscatter model for the hhvv coherence phase which we adapt from the past frameworks by [13], [16]. We use this to forward model the azimuth and phase symmetry of the (deterministic) 'co-polarized hhvv crossterm' which we relate to the (stochastic) hhvv coherence. The model-data comparison enables us to demonstrate how the azimuth and depth-dependence of the vertical gradient of the hhvv coherence phase can be used to determine COF properties. Specifically, under the assumption that one of the fabric eigenvectors is vertical, we can determine the depthdependence of the dielectric principal axes (related to the horizontal eigenvectors of the orientation tensor and the prevailing crystallographic axis in the horizontal plane), and the birefringence (related to the difference between the horizontal eigenvalues and a measure of horizontal fabric asymmetry).

The radar-inferred COF eigenvalue difference is compared with the NEEM ice core COF data [5] providing the first field data validation for the polarimetric coherence method. The results conform to a conventional dynamical model of deformation at an ice divide where a lateral component of tension is present [3], [9]. In particular, at mid-depths we infer horizontal asymmetry to the COF and the greatest horizontal concentration of $c$-axes orientated near-parallel to the ice divide.

\section{DiELECTRIC ANISOTROPY OF POLAR ICE}

\section{A. Dielectric anisotropy of individual ice crystals}

Individual ice crystals have hexagonal structure and are uniaxially birefringent with the optic axis aligned with the crystallographic axis ( $c$ axis) [12]. The crystal birefringence is notated by $\Delta \epsilon^{\prime}=\left(\epsilon_{\| c}-\epsilon_{\perp c}\right)$ where $\epsilon_{\| c}$ and $\epsilon_{\perp c}$ are the principal permittivities parallel and perpendicular to the $c$-axis [24]. At radar sounding frequencies $(\sim 50-400 \mathrm{MHz})$, and as ice temperature increases from $-60-0{ }^{\circ} \mathrm{C}, \epsilon_{\perp c}$ and $\epsilon_{\| c}$ increase from $\sim 3.12-3.16$ and $\Delta \epsilon^{\prime}$ increases by $\sim 5 \%$ from $\sim 0.0325-0.0345$ [13], [24], [25]. In the data analysis in this study, following [13], we assume $\left(\epsilon_{\| c}-\epsilon_{\perp c}\right)=0.034$.

\section{B. Dielectric anisotropy of the COF}

Polar ice sheets are polycrystalline and the probability distribution of $c$-axis orientation statistically quantifies the COF. A second order orientation tensor and the corresponding set of normalized eigenvalues $\left(E_{1}+E_{2}+E_{3}=1\right)$ and eigenvectors can be used to describe COF anisotropy [7]. Each eigenvalue describes the relative concentration of $c$-axes aligned in each principal direction with higher eigenvalues indicating greater concentrations. In this study we assume the convention $E_{3}>E_{2}>E_{1}$ which is done for consistency with past radar polarimetry studies [2], [3], [13]. It should, however, be noted that the opposite convention $\left(E_{3}<E_{2}<E_{1}\right)$ is sometimes used in ice core studies (e.g. [5]). A range of endmember categories are used to describe COF distributions and include: 'random fabrics' where $E_{1} \approx E_{2} \approx E_{3} \approx \frac{1}{3}$ (typically associated with the near-surface), 'single-pole fabrics' where $E_{1} \approx E_{2} \approx 0, E_{3} \approx 1$ (typically associated with deep ice undergoing vertical compression), and 'vertical girdle fabrics' where $E_{1} \approx 0, E_{2} \approx \frac{1}{2}, E_{3} \approx \frac{1}{2}$ (typically associated with horizontal tension at moderate ice depths) [3], [5]. Visual examples of different COF distributions are provided in [26], [27].

Individual ice crystals have dimensions $\sim \mathrm{mm}$, approximately two orders of magnitude smaller than the radio wavelength in ice ( $\sim 1-5 \mathrm{~m}$ for most ice-penetrating radar systems). It is therefore the macroscopic bulk birefringence of the COF (which in this study we also refer to as 'fabric birefringence') that determines the polarimetric response of radio waves in polar ice [12]. In the general case, where $E_{1} \neq E_{2} \neq E_{3}$, the COF behaves as a biaxial media, with three different principal permittivites [13]. The principal coordinate system of the fabric orientation tensor, notated using $(x, y, z)$ or $(1,2,3)$, is in general different from the coordinates used in mono-static nadir radar sounding measurements, notated using $\left(x^{\prime}, y^{\prime}, z^{\prime}\right)$. However, typically the fabric eigenvector with the largest eigenvalue can be approximated as vertical (i.e. $z=z^{\prime}$ ), with the other two eigenvectors in the horizontal plane [13], [28], [29] and we use this approximation throughout the majority of this study. Physically this scenario arises due to compaction of the firn and the dominance of vertical compression. 
Following a geometric argument, the principal dielectric tensor of the COF can be expressed as

$$
\begin{aligned}
\underline{\underline{\epsilon}} & =\left(\begin{array}{ccc}
\epsilon_{x} & 0 & 0 \\
0 & \epsilon_{y} & 0 \\
0 & 0 & \epsilon_{z}
\end{array}\right), \\
& =\left(\begin{array}{ccc}
\epsilon_{\perp c}+E_{1} \Delta \epsilon^{\prime} & 0 & 0 \\
0 & \epsilon_{\perp c}+E_{2} \Delta \epsilon^{\prime} & 0 \\
0 & 0 & \epsilon_{\perp c}+E_{3} \Delta \epsilon^{\prime}
\end{array}\right),
\end{aligned}
$$

[13] and therefore the bulk dielectric properties of anisotropic polar ice can be described in terms of the birefringence of an individual ice crystal and the eigenvalues of the fabric orientation tensor. For nadir measurements the transversely polarized radio wave is sensitive to the fabric birefringence in the horizontal plane, which from (1) is given by

$$
\Delta \epsilon=\epsilon_{y}-\epsilon_{x}=\Delta \epsilon^{\prime}\left(E_{2}-E_{1}\right)
$$

where $\left(E_{2}-E_{1}\right)$ quantifies the horizontal asymmetry of the fabric, or equivalently the strength of the vertical girdle. The presence of a tilt angle between the $E_{3}$ eigenvector and the vertical direction is an additional source of horizontal birefringence [2] and Appendix A provides a generalization of (2) that incorporates this rotation.

\section{POLARIMETRIC MATRIX BACKSCATTER MODEL}

\section{A. Model overview}

The matrix backscatter model in this study is based upon the formulation by [13] which is similar to a previous formulation by [16]. The model considers a nadir sounding geometry where the ice sheet is modeled as a stratified anisotropic medium and can be used to model biaxial fabric under the previously discussed assumption that one of the dielectric principal axes/fabric eigenvectors is vertical. The dielectric properties of each layer are specified by the strength of the fabric birefringence, (2), and the azimuthal orientation of the dielectric principal axes.

The model coordinate systems are shown in Fig. 1(a) where $(x, y)$ is the principal axis system and $\left(x^{\prime}, y^{\prime}\right)$ is the measurement system (coincident with $h$ and $v$ polarization planes) and $\alpha$ is the azimuthal rotation angle between the principal axis system and the measurement system. The bearing $\theta$, used to georeference the data in Sect. $\mathrm{V}$, is also indicated. The $h$ and $v$ polarization planes are therefore both aligned with the ice-sheet surface, and we assume the convention that when $\alpha=0^{\circ}$ the $v$ polarization is aligned with greatest principal permittivity, $\epsilon_{y}$.

The model considers a specific scenario of electromagnetic propagation in biaxial media: when the propagation direction is aligned with one of the principal axes (the vertical). This scenario can be visualized from the 2D biaxial indicatrix (refractive index surface) section in Fig. 1(b), and a detailed discussion of this representation in radar sounding is provided by [30]. The semi-major and semi-minor (principal) axes have refractive indices $n_{x}=\sqrt{\epsilon}_{x}$ and $n_{y}=\sqrt{\epsilon}_{y}$, and linearly polarized measurements orientated with these axes $\left(\alpha=0,90^{\circ}\right)$ propagate at phase velocities of $c / n_{x}$ (the fast axis) and $c / n_{y}$ (the slow axis) respectively. Linearly polarized measurements (a) Coordinates

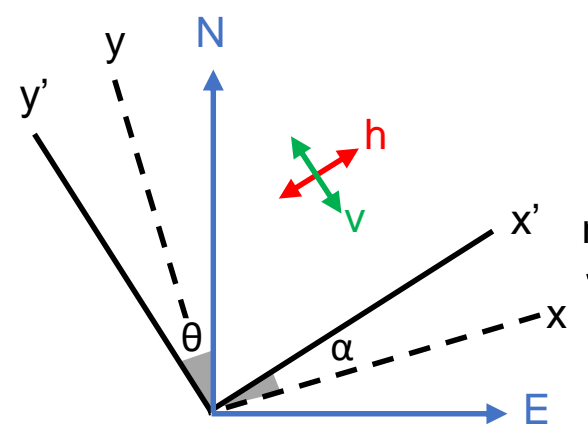

(b) Indicatrix section

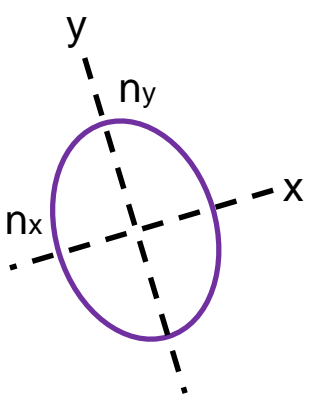

Fig. 1. (a) Model coordinate systems. $(x, y)$ is the principal axis system, $\left(x^{\prime}, y^{\prime}\right)$ is the measurement system (coincident with $h$ and $v$ polarization planes) and is orientated at azimuthal rotation angle $\alpha$ to $(x, y) . \theta$ is the bearing of the $y^{\prime}$ axis ( $v$ polarization plane) relative to due north. (b) Crosssection of biaxial indicatrix (refractive index surface) with exaggerated aspect ratio.

not orientated with the principal axes split into a superposition of these two principal wave components as they propagate through the ice sheet.

The model physics incorporates the combined effect of birefringent propagation (transmission through anisotropic layers) and anisotropic scattering (reflection from the interface between two different anisotropic layers). Radar reflections originate from three different physical mechanisms: density contrasts (at shallow depths only), conductivity contrasts, and COF contrasts [31]. Only COF contrasts can be anisotropic (i.e. the reflection strength depends upon polarization). The layer interfaces are treated as coherent scatters (i.e. scattering is equivalent to a specular reflection) and the model neglects multiple scattering and interference. Both the matrix model simulations and the model-data comparison assume that the radar pulse is monochromatic (i.e. we neglect the effects of the finite bandwidth).

The matrix model has previously been used to simulate the azimuth and phase/depth dependence of polarimetric power anomalies resulting from either birefringent propagation, anisotropic scattering or their combined effect [2], [13]. The original contribution in this study is to simulate the azimuthal properties of the deterministic "co-polarized hhvv cross-term', $s_{h h v v}$, which has analogous phase behavior to the stochastic $h h v v$ complex coherence, $c_{h h v v}$, (explained in more detail in Sect. III-C).

\section{B. Scattering matrix decomposition}

In the scattering model the electric field components are modelled as harmonic plane waves and in the principal axis coordinate system, $(x, y)$, are of the form:

$$
\begin{aligned}
& E_{x}=E_{x 0} \exp [i(k z-\omega t+\delta)], \\
& E_{y}=E_{y 0} \exp [i(k z-\omega t)],
\end{aligned}
$$

where $E_{x 0}$ and $E_{y 0}$ are field amplitudes, $k$ is the wave number, $\omega$ is the angular frequency, $\delta$ is the two-way phase shift (for backwards and forwards propagation in the ice sheet) 
and $t$ is time [13], [16]. Inverse square spreading losses do not affect the polarimetric response and the electric field can be modeled as a normalized Jones vector of the form $\mathbf{E}=$ $\left(E_{x 0} \exp (i \delta), E_{y 0}\right)^{T}$ where the common $\exp [(i(k z-\omega t)]$ factor in (3) and (4) is removed [16] and $T$ notates transpose.

The radar measurements are made in the $\left(x^{\prime}, y^{\prime}\right)$ coordinate system with electric field components given by $\mathbf{E}_{R}=$ $\left(E_{h}, E_{v}\right)_{R}^{T}$ and $\mathbf{E}_{\tau}=\left(E_{h}, E_{v}\right)_{\tau}^{T}$ where the subscripts $R$ and $\tau$ notate received and transmitted [16]. The polarimetric backscattering processes are given by the general matrix equation

$$
\mathbf{E}_{R}=S \mathbf{E}_{\tau}
$$

where

$$
S=\left(\begin{array}{ll}
s_{h h} & s_{h v} \\
s_{v h} & s_{v v}
\end{array}\right),
$$

is the scattering matrix, where matrix elements $s_{h h}$ and $s_{v v}$ in (6) indicate co-polarized scattering amplitudes and $s_{v h}$ and $s_{h v}$ indicate cross-polarized. Only $s_{h h}$ and $s_{v v}$ are relevant for the co-polarized analysis method in this study.

$S$ is decomposed into three physical processes: forward propagation/transmission, scattering/reflection and backward propagation. Following past applications [2], [13], we consider a single, depth-invariant, principal axes orientation for the scattering and the propagation layers. This results in the following matrix decomposition

$$
S(\alpha, \delta, r)=R(\alpha) P(\delta / 2) \Gamma(r) P(\delta / 2) R^{\prime}(\alpha),
$$

where

$$
R(\alpha)=\left(\begin{array}{cc}
\cos (\alpha) & \sin (\alpha) \\
-\sin (\alpha) & \cos (\alpha)
\end{array}\right)
$$

is the $2 \mathrm{D}$ rotation matrix, $R^{\prime}(\alpha)=R^{T}(\alpha)$ is the inverse 2D rotation matrix,

$$
P(\delta / 2)=\left(\begin{array}{cc}
\exp (i \delta / 2) & 0 \\
0 & 1
\end{array}\right)
$$

is the propagation/transmission matrix, and

$$
\Gamma(r)=\left(\begin{array}{ll}
r & 0 \\
0 & 1
\end{array}\right)
$$

is the reflection matrix with $r$ the ratio of the (E-field) Fresnel reflection coefficient along the principal axes. The choice of notation in (7) largely follows the presentation by [16].

For the single principal-axes orientation considered here, (7) applies at each reflecting layer. The two-way phase shift, $\delta$, increases with ice depth and is given by

$$
\delta=\delta_{x}-\delta_{y}=\frac{4 \pi f}{c} \int_{z_{0}}^{z}\left(\sqrt{\epsilon_{y}(z)}-\sqrt{\epsilon_{x}(z)}\right) d z
$$

where $f$ is the center frequency, $c$ is the vacuum speed of the radio wave, $z_{0}$ is an initial depth [13]. Following a first order Taylor expansion, for small deviations about a mean permittivity, (11) can be expressed as

$$
\delta=\frac{4 \pi f}{c} \int_{z_{0}}^{z} \frac{\Delta \epsilon(z)}{2 \sqrt{\bar{\epsilon}}} d z
$$

where $\bar{\epsilon}$ is the mean (polarization-averaged) permittivity (assumed here to be a constant $\bar{\epsilon}=3.15$ ).
There are three degrees of freedom in (7): $\alpha, \delta$ and $r$. The assumption that the dielectric principlal axes are the same for scattering and propagation layers accommodates the situation when the strength of a (non-ideal) vertical singlepole $\operatorname{COF}\left(E_{3}>>E_{2} \approx E_{1}\right)$ or (non-ideal) vertical girdle $\left(E_{1}<<E_{2} \approx E_{3}\right)$ change with depth [13]. The model-data comparison in this study shows that a three parameter model is generally sufficient to understand radar-inferred fabric properties at NEEM. However, to illustrate the generality of the coherence method, Appendix B extends the scattering model to consider a principal axes rotation with ice depth.

\section{Modelling the hhvv cross-term, $s_{h h v v}$}

The data analysis in this study, Sect. IV-B and Sect. V, utilizes the hhvv coherence to quantify the phase correlation between $s_{h h}$ and $s_{v v}$ where $h h$ and $v v$ are two co-polarized measurements that differ by a rotation of 90 degrees in the horizontal plane. The hhvv coherence is a stochastic parameter defined by

$$
c_{h h v v}=\frac{<s_{h h} \cdot s_{v v}^{*}>}{\sqrt{<\left|s_{h h}\right|^{2}>} \sqrt{<\left|s_{v v}\right|^{2}>}},
$$

where $\langle\ldots\rangle$ notates expectation value and * indicates complex conjugate [18]. $c_{h h v v}$ is a complex number defined within the unit circle. The coherence magnitude, $\left|c_{h h v v}\right|$, quantifies the correlation strength between $h h$ and $v v$ measurements and is defined on the interval $[0,1]$ with 1 representing a perfect correlation. In this study, the ensemble averages in (13) are evaluated over a vertical averaging cell of length $\sim 100 \mathrm{~m}$ (see Sect. IV-B for more details). The hhvv coherence phase,

$$
\phi_{h h v v}=\arg \left(c_{h h v v}\right),
$$

provides a statistically efficient estimate of the phase difference between the $h h$ and $v v$ measurements and is also referred to as the co-polar or co-polarized phase difference [32].

By using the matrix backscatter model to calculate $s_{h h}$ and $s_{v v}$ we can consider an analogue to (13):

$$
s_{h h v v}=\frac{s_{h h} \cdot s_{v v}^{*}}{\sqrt{\left|s_{h h}\right|^{2}} \sqrt{\left|s_{v v}\right|^{2}}},
$$

where the expectation values in (13) are replaced with deterministic simulated values. $s_{h h v v}$ - which we refer to as the 'hhvv cross-term' - is a complex number defined on the circumference of the unit circle. Therefore $\left|s_{h h v v}\right|=1$ and $\left|c_{h h v v}\right|$ is not directly comparable to $\left|s_{h h v v}\right|$. However, for the model-data comparison in this study we assume that the hhvv coherence phase is comparable to the modeled hhvv phase difference, i.e.: $\phi_{h h v v}=\arg \left(c_{h h v v}\right)=\arg \left(s_{h h v v}\right)$.

For depth-invariant principal axes $s_{h h v v}$ can be numerically evaluated using (7) or analytically, which aids in the physical interpretation of the simulations in the next sections. To evaluate $s_{h h v v}$ analytically we first calculated the co-polarized complex amplitudes from (7) which are given by

$$
\begin{aligned}
& s_{h h}=r \cos ^{2}(\alpha) \exp (i \delta)+\sin ^{2}(\alpha) \\
& s_{v v}=\cos ^{2}(\alpha)+r \sin ^{2}(\alpha) \exp (i \delta) .
\end{aligned}
$$




\section{Isotropic scattering}
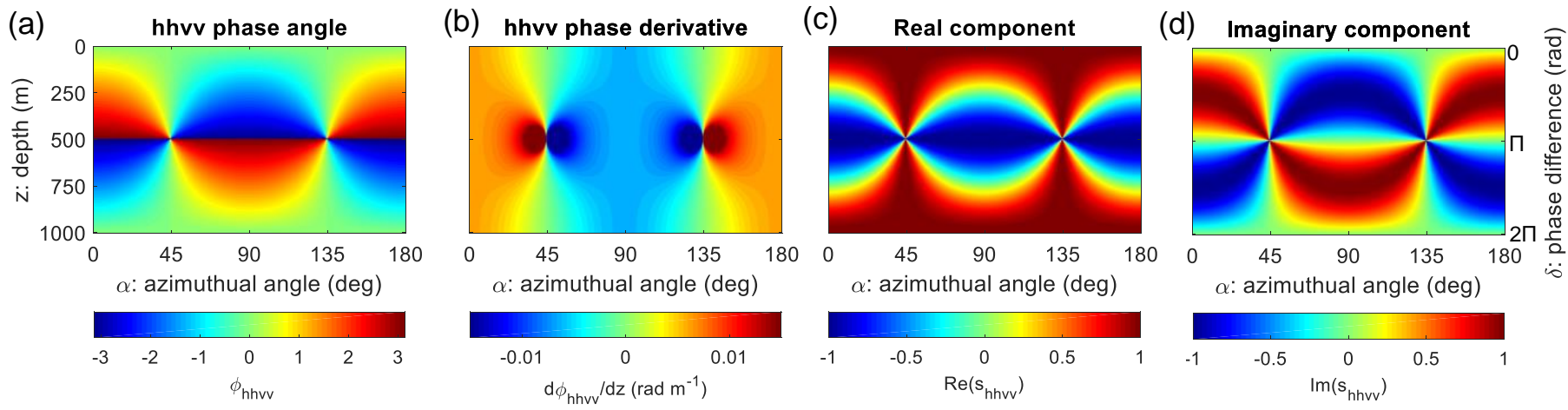

Fig. 2. Matrix backscatter model results for $s_{h h v v}$ with isotropic scattering $(r=1)$. (a) hhvv phase, $\phi_{h h v v}=\arg \left(s_{h h v v}\right)$. (b) Vertical $h h v v$ phase gradient, $d \phi_{h h v v} / d z$. (c) Real component, $\operatorname{Re}\left(s_{h h v v}\right)$, (d) Imaginary component, $\operatorname{Im}\left(s_{h h v v}\right)$. The relationship between $z$ and $\delta$ assumes a two-way phase shift following (18) with a fixed fabric birefringence of $\Delta \epsilon=0.00354$ and radar center frequency, $f=150 \mathrm{MHz}$. When $\alpha=0^{\circ}$ the $v$ polarization is aligned with the greatest principal permittivity resulting in a positive $h h v v$ phase gradient.

It follows that

$$
\begin{aligned}
s_{h h} . s_{v v}^{*} & =r\left(\cos ^{4}(\alpha) \exp (i \delta)+\sin ^{4}(\alpha) \exp (-i \delta)\right) \\
& +\left(1+r^{2}\right) \cos ^{2}(\alpha) \sin ^{2}(\alpha),
\end{aligned}
$$

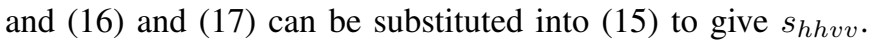

\section{Model results for isotropic scattering and constant bire- fringence}

Past radar sounding studies indicate that birefringent propagation combined with isotropic scattering can be a useful approximation for certain fabric regimes [2], [14]. We therefore initially consider evaluating $s_{h h v v}$ for the case of isotropic scattering, $(r=1)$. In this example we also consider constant fabric birefringence and constant mean permittivity with ice depth which, from (12), results in a linear relationship between $\delta$ and $z$ of the form

$$
\delta=\frac{4 \pi f}{c} \frac{\Delta \epsilon}{2 \sqrt{\epsilon}}\left(z-z_{0}\right)+\delta_{0}
$$

where $\delta_{0}$ is a reference phase. To make a connection with the later data analysis we assume $\Delta \epsilon=0.00354 \approx 0.1 \Delta \epsilon^{\prime}$ (a fabric birefringence order a tenth of the single crystal birefringence). This value is chosen so that a $2 \pi$ phase period occurs over a $1000 \mathrm{~m}$ depth interval, and is broadly comparable to the fabric birefringence we infer at NEEM. This step enables us to plot $d \phi_{h h v v} / d z$ (the vertical $h h v v$ phase derivative) which is later used to determine fabric orientation and asymmetry. The real and imaginary components of $s_{h h v v}, \operatorname{Re}\left(s_{h h v v}\right)$ and $\operatorname{Im}\left(s_{h h v v}\right)$, are used in the numerical evaluation of the (data) phase derivative, Sect. IV-C, and provide a useful tool to further visualize the azimuth and phase/depth symmetry.

The azimuth-phase $(\alpha, \delta)$ dependence for: $\phi_{h h v v}$, $d \phi_{h h v v} / d z, \operatorname{Re}\left(s_{h h v v}\right), \operatorname{Im}\left(s_{h h v v}\right)$ are shown in Fig. 2. $\phi_{h h v v}$ and $d \phi_{h h v v} / d z$ both have 180 degree periodicity, with 90 degree 'azimuthal zones' where there are positive and negative phase gradients. Additionally, $\phi_{h h v v}$ and $d \phi_{h h v v} / d z$ both have planes of mirror symmetry about $\alpha=0,90^{\circ}$. Along the principal axes $\left(\alpha=0,90^{\circ}\right), d \phi_{h h v v} / d z$ is constant with $\delta$. There is asymptotic behavior in $d \phi_{h h v v} / d z$ as $\alpha$ approaches $45,135^{\circ}$ and $\delta$ approaches $\pi$ radians. $\operatorname{Re}\left(s_{h h v v}\right)$ and $\operatorname{Im}\left(s_{h h v v}\right)$ have azimuthal periodicity of 90 and 180 degrees respectively. $\operatorname{Re}\left(s_{h h v v}\right)$ has planes of mirror symmetry about $\alpha=0,45,90,135^{\circ}$ and $\operatorname{Im}\left(s_{h h v v}\right)$ has planes of mirror symmetry about $\alpha=0,90^{\circ}$.

The results in Fig. 2 can be understood from (17) as $\phi_{h h v v}$ is governed by the numerator of (13), $s_{h h} . s_{v v}^{*}$. In particular, (17) indicates that $s_{h h} . s_{v v}^{*}=\exp (i \delta)$ when $\alpha=0^{\circ}$ (i.e. there is a positive phase shift with increasing depth), whereas $s_{h h} . s_{v v}^{*}=\exp (-i \delta)$ when $\alpha=90^{\circ}$ (i.e. there is negative phase shift with increasing ice depth). This sign convention occurs because, we previously defined $\epsilon_{y}>\epsilon_{x}$ (i.e. the $x$ axis is the fast axis). For other values of $\alpha$, (17) indicates that $s_{h h v v}$ comprises a superposition of trigonometrically-weighted positive and negative phase shifts.

The results in Fig. 2 can be compared with scattering model simulations for co-polarized power in [13][Fig. 5(a)] (two phase periods) and [2][Fig. 7(a)] (a single phase period as is considered here). A key result is that the co-polarized nodes occur at the same point the asymptotes in $d \phi_{h h v v} / d z$ do: $\alpha=$ $45,135^{\circ}$ and $\delta=\pi$ radians.

\section{E. Model results for anisotropic scattering and constant bire- fringence}

To assess the robustness of the coherence method for different layer scattering regimes we now consider the effect of anisotropic scattering upon the periodicity of $\phi_{h h v v}$ and $d \phi_{h h v v} / d z$. We will also later demonstrate in Sect. V that the inclusion of anisotropic scattering $(r \neq 1)$ is necessary to explain the observed properties of $\phi_{h h v v}$ for the MCRDs data set at NEEM. To assess the effect of anisotropic scattering we considered the same scenarios that [2][Fig. 7(c,d)] did for polarimetric power anomalies, corresponding to $5 \mathrm{~dB}$ and 10 $\mathrm{dB}$ perturbations to the power reflection coefficient (equivalent to setting $r=10^{\frac{1}{4}}$ and $r=10^{\frac{1}{2}}$ in (10)). As in [2], these simulations correspond to the case that the principal axes of the scattering layer are aligned with the propagation layer.

Model results for anisotropic scattering are shown in Fig. 3 that use the same constant birefringence as isotropic scattering 

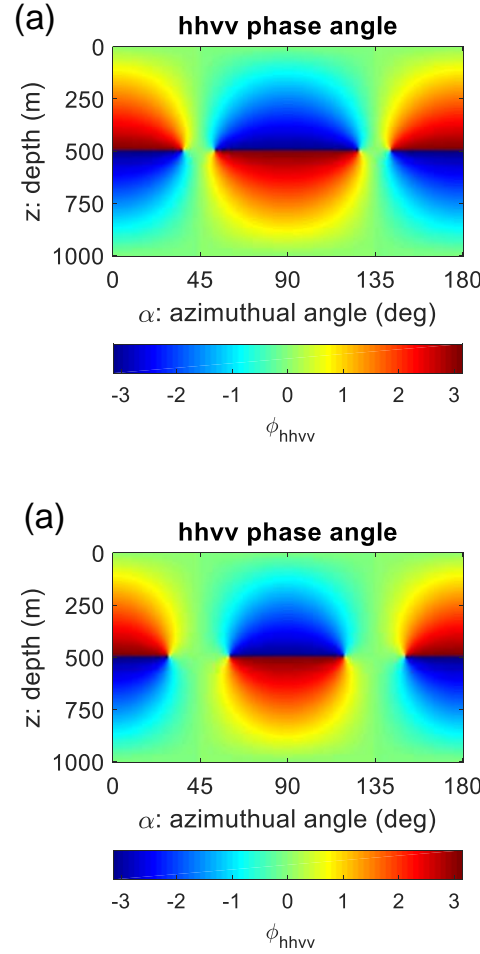

Anisotropic scattering ( $5 \mathrm{~dB})$

(b) hhvv phase derivative

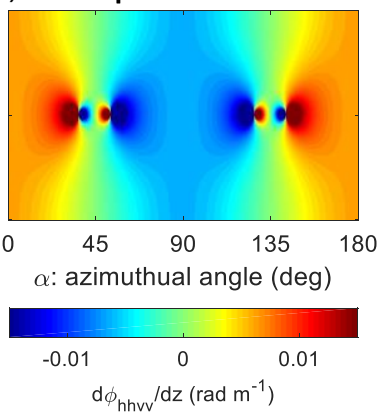

Anisotropic scattering (10 dB)

(b) hhvv phase derivative

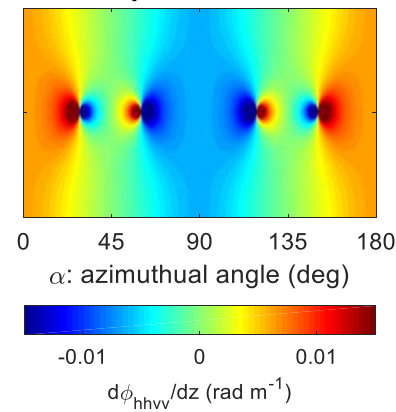

(c)

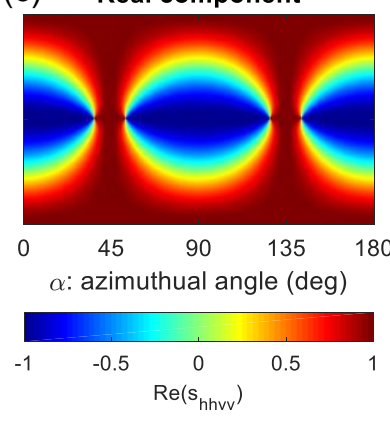

(d)

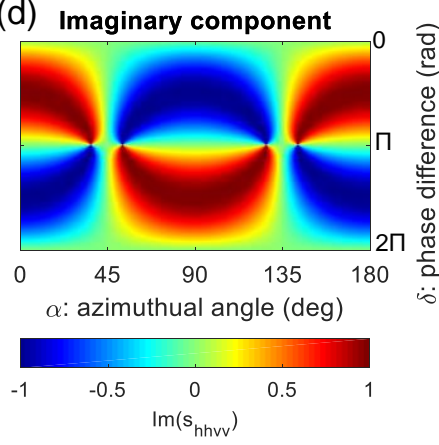

0

(c) Real component

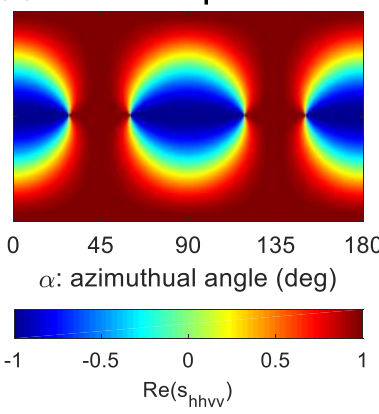

(d) Imaginary component

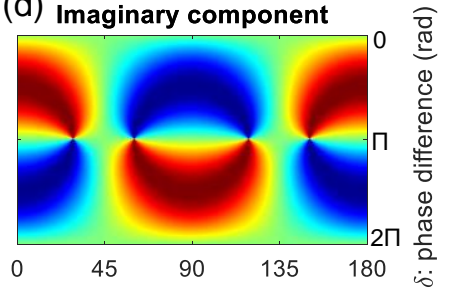

$\alpha$ : azimuthual angle (deg)

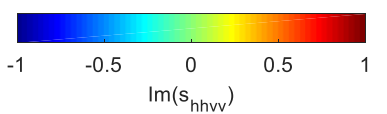

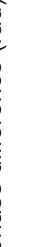

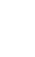

Fig. 3. Matrix backscatter model results for $s_{h h v v}$ with anisotropic scattering for $5 \mathrm{~dB}\left(r=10^{\frac{1}{4}}\right)$ and $10 \mathrm{~dB}\left(r=10^{\frac{1}{2}}\right)$. (a) $h h v v$ phase, $\phi_{h h v v}=\arg \left(s_{h h v v}\right)$. (b) Vertical $h h v v$ phase gradient, $d \phi_{h h v v} / d z$. (c) Real component, $\operatorname{Re}\left(s_{h h v v}\right)$, (d) Imaginary component, Im( $\left.s_{h h v v}\right)$. The relationship between $z$ and $\delta$ assumes a two-way phase shift following (18) with a fixed fabric birefringence of $\Delta \epsilon=0.00354$ and radar center frequency, $f=150 \mathrm{MHz}$.

in Fig. 2. The inclusion of anisotropic scattering results in the removal of the strict 90 degree azimuthal zones where there are positive and negative phase gradients, with locally alternating zones of positive and negative phase gradients near to $\alpha=45,135^{\circ}$ present. The azimuthal periodicity - 180 degree for $\phi_{h h v v}, d \phi_{h h v v} / d z$, and $\operatorname{Im}\left(s_{h h v v}\right)$ and 90 degree for $\operatorname{Re}\left(s_{h h v v}\right)$ - and planes of mirror symmetry - $\alpha=0,45$, $90,135^{\circ}$ for $\phi_{h h v v}, d \phi_{h h v v} / d z$, and $\operatorname{Im}\left(s_{h h v v}\right)$ and $\alpha=0,90$ $\circ$ for $\operatorname{Re}\left(s_{h h v v}\right)$ - are, however, preserved from the isotropic case. Notably, in Fig. 3(b) the azimuthal center of the 'zones' of positive and negative hhvv phase gradient are still aligned with the principal axis.

\section{DATA ANALYSIS METHODOLOGY}

In this study we consider a data analysis methodology for calculating the polarimetric coherence that applies to groundbased, single-polarized, radar sounding measurements as a function of azimuthal angle. This multi-polarization method applies to both 'turning circle' measurements where an antenna is driven in a circular track (the case described here for the MCRDs data set) or 'pirouette' measurements when transmit and receive antenna are co-rotated at a fixed location [2], [3], [13], [15]. Due to the 180 degree azimuthal periodicity of the model predictions the coherence method can determine fabric properties from 180 degree periodic data (as is the case for pirouette measurements). Here, however, we use 360 degree periodic data to illustrate the reproducibility of the method.

\section{A. Radar system and field site}

The radar measurements used to demonstrate the method are from a ground-survey using the MCRDS (Multi Channel Radar Depth Sounder) radar developed by CReSIS (Center for Remote Sensing of Ice Sheets). The MCRDS radar is described in detail by [14] including a table of the system parameters. The center frequency is $150 \mathrm{MHz}$ with a $30 \mathrm{MHz}$ bandwidth, whilst the depth-range resolution in ice is $\sim 2.8 \mathrm{~m}$ which matches the depth-range sample spacing. The radar data processing is as described in [14] 2018 (including reduction of speckle artifacts).

The radar data were collected from the NEEM ice core region in northwest Greenland in August 2008 prior to drilling of the core, Fig. 4. As part of a more extensive survey grid, radar measurements were made in three complete turning circles - labeled as $A, B$, and $C$ - and centered at latitude-longitude coordinates of $(77.405,-51.171)^{\circ},(77.463$, $-51.261)^{\circ},(77.484,-50.967)^{\circ}$ with ice thicknesses of 2517 $\mathrm{m}, 2497 \mathrm{~m}, 2542 \mathrm{~m}$ respectively. The diameter of the circles is $\sim 50 \mathrm{~m}$. In the previous analysis by [14] measurements to the southeast of the ice core with incomplete azimuthal data were also considered, and the labels $A, B$ and $C$ in our study correspond to 1,3 and 4 in their study.

The full data set described by [14] comprises of quadpolarized measurements and single-polarized measurements. The single-polarized measurements are equivalent to copolarized ( $h h$ or $v v$ ) measurements and the polarization plane is aligned with the track direction. In this study, with a view 

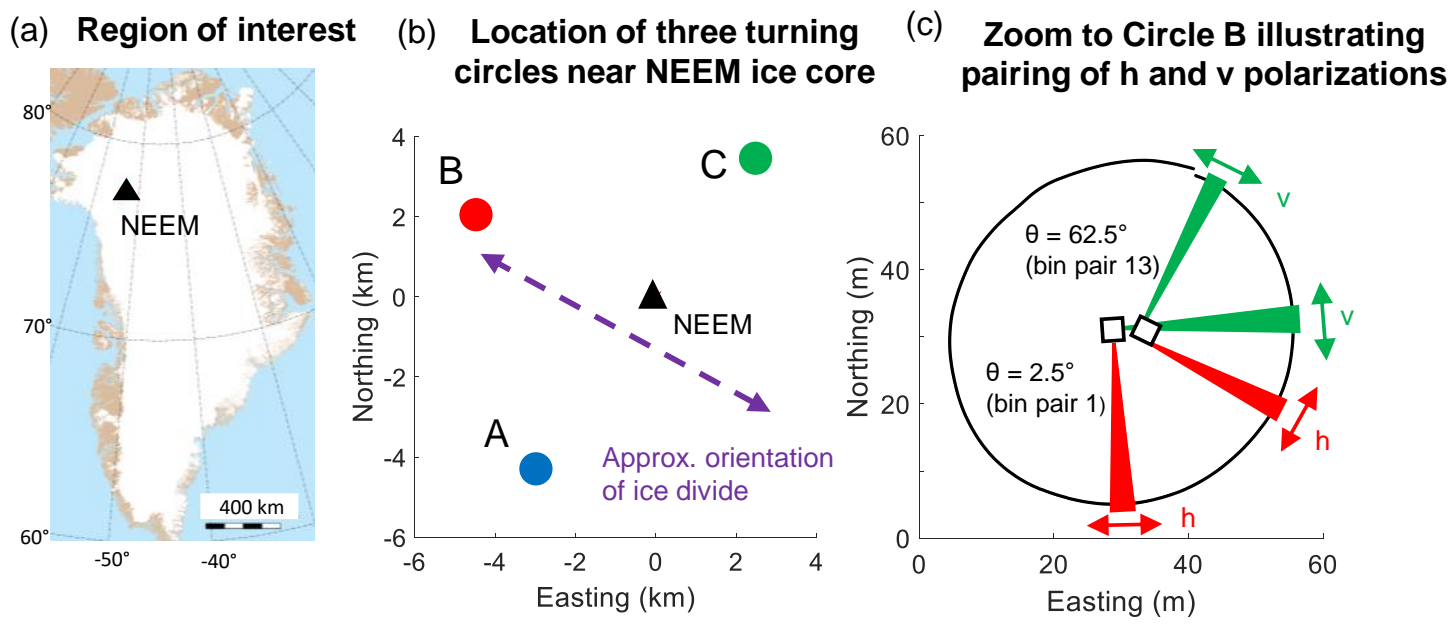

Fig. 4. (a) Region of interest. (b) Location of the three turning circles relative to NEEM ice core and approximate orientation of ice divide (approximately parallel to $\theta=60^{\circ}$ ). (c) Example zoom to site $B$. The polarization plane is aligned with the track direction and $h h$ - $v v$ polarization pairs as a function of azimuth are generated by rotating the bin configurations in (c) counter-clockwise. The first bin configuration for $h$ and $v$ polarizations is indicated (bin centers at $\theta=2.5^{\circ}$ ), along with the bin configuration for when the $v$ polarization plane is aligned approximately parallel to the ice divide (bin center at $\theta=62.5^{\circ}$ ). The $5^{\circ}$ angular bin widths are indicated by the red and green segments and the white squares indicate that the polarization planes in each $h h-v v$ pair are orthogonal.

toward being able to reconstruct information from a nonpolarimetric radar system, we focus purely upon the singlepolarized measurements and exploit varying track/antenna orientation to obtain orthogonal polarizations. We define $h$ and $v$ polarizations in relation to the bearing $\theta$ and $x^{\prime}$ and $y^{\prime}$ axes (see Fig. 1). When $\theta=0^{\circ}$ the $h$ polarization is therefore aligned with the east/west axis, and the $v$ polarization is aligned with the north/south axis. For counter-clockwise rotation $h$ therefore lags $v$ by 90 degrees, and Fig. 4(c) shows examples of $h h-v v$ azimuth bin pairs. We used an angular bin size of $5^{\circ}$ which corresponds to a mean bin counts of 13.4, 8.8, 9.3 for sites $A, B$ and $C$ respectively.

The definition of $h$ and $v$ in this study differs from satellite SAR polarimetry where $h$ is conventionally aligned with the along-track direction and is intended to make the simplest connection with the scattering model geometry, Fig. 1.

\section{B. Numerical evaluation of hhvv coherence, $c_{h h v v}$}

For each $h h-v v$ bin pair in Fig. 4(c) the hhvv coherence, (13), can be estimated via the discrete approximation

$$
c_{h h v v}=\frac{\sum_{i=1}^{N} s_{h h, i} \cdot s_{v v, i}^{*}}{\sqrt{\sum_{i=1}^{N}\left|s_{h h, i}\right|^{2}} \sqrt{\sum_{i=1}^{N}\left|s_{v v, i}\right|^{2}}},
$$

where $N$ is the number of independent samples and $i$ is a summation index [20], [33]. In satellite polarimetry applications, (19) is assessed over a 2D horizontal cell (e.g. [17]). In radar-sounding applications, (19) can be assessed using either azimuthal/horizontal or range/vertical averaging [20]. Averaging in range ensures that there are $\sim N$ independent samples when applying (19) as the depth-range sample spacing of the MCRDs data set is approximately the same as the range resolution. However, we do not apply (19) over azimuth, as the radar measurements are only separated by a few $\mathrm{m}$, and, based upon Fresnel zone dimensions, are not spatially independent. Instead, to improve the SNR, we first coherently averaged the $h h$ and $v v$ measurements in each azimuth bin and then apply (19) over the range dimension. This approach implicitly assumes that, for coherent combination, the ice interfaces act as horizontal specular reflectors.

As stated in Sect. III-C, $\left|c_{h h v v}\right|$ quantifies the correlation strength between $h h$ and $v v$ and is defined on the interval $[0,1]$. Based upon analogy with the interferometric phase and the statistics of coherence estimation [33], the Cramer-Rao bound can be used to estimate a phase error via

$$
\sigma_{\phi h h v v} \approx \frac{1}{\left|c_{h h v v}\right|} \sqrt{\frac{1-\left|c_{h h v v}\right|^{2}}{2 N}}
$$

In the data analysis we used a sliding window of $\sim 100 \mathrm{~m}$ in length (corresponding to $N=36$ ). We limit data interpretation to sections of the ice column where the angular average of $\left|c_{h h v v}\right|>0.4$, which from (20) corresponds to an error $\sigma_{\phi_{h h v v}}$ $<0.27$ radians. The choice of window size ensures that $N$ is sufficiently large to provide an unbiased estimate of $c_{h h v v}$ (see [33]), whilst being of a suitable resolution to compare with ice core COF data (see Sect. V-D).

The coherence phase, $\phi_{h h v v}=\arg \left(c_{h h v v}\right)$, is defined up to an arbitrary phase shift (equivalent to multiplication of $c_{h h v v}$ by a phase term $\exp \left(i \phi_{0}\right)$ where $\phi_{0}$ is a reference phase). In order to align the relative phases from the different bin pairs we used the property that $\phi_{h h v v}(\alpha, \delta=\pi)=\pi$ for all values of $\alpha$ (see Figs. 2 and 3). It is, however, important to note that the primary diagnostic for COF properties, $d \phi_{h h v v} / d z$, is independent of $\phi_{0}$. 
C. hhvv phase gradient method to determine fabric birefringence and asymmetry

The previous analysis by [20] used the absolute value of the $h h v v$ phase gradient, $\left|d \phi_{h h v v} / d z\right|$, to determine the birefringence and the horizontal asymmetry of the COF. In their analysis [20] considered the case where the $h$ and $v$ polarization planes were assumed to be aligned with the principal axes system $\left(\alpha=0,90^{\circ}\right)$ and $\left|\phi_{h h v v}\right|=\delta$ (i.e. the $h h v v$ phase difference is equivalent to the principal axes phase retardation). In this scenario it follows from (12) that

$$
\left|\frac{d \phi_{h h v v}\left(\alpha=0,90^{\circ}\right)}{d z}\right|=\frac{d \delta}{d z}=\frac{4 \pi f}{c} \frac{\Delta \epsilon(z)}{2 \sqrt{\bar{\epsilon}}},
$$

which, using (2), can be expressed in terms of the orientational eigenvalues as

$$
\left|\frac{d \phi_{h h v v}\left(\alpha=0,90^{\circ}\right)}{d z}\right|=\frac{d \delta}{d z}=\frac{4 \pi f}{c} \frac{\Delta \epsilon^{\prime}\left(E_{2}(z)-E_{1}(z)\right)}{2 \sqrt{\bar{\epsilon}}} .
$$

Hence the fabric asymmetry, $E_{2}-E_{1}$, and fabric birefringence, $\Delta \epsilon$, are both proportional to $\left|d \phi_{h h v v}\left(\alpha=0,90^{\circ}\right) / d z\right|$.

To evaluate $d \phi_{h h v v} / d z$ the following identity can be used

$$
\frac{d \phi_{h h v v}}{d z}=\frac{R \frac{d I}{d z}-I \frac{d R}{d z}}{R^{2}+I^{2}}
$$

where $R=\operatorname{Re}\left(c_{h h v v}\right)$ and $I=\operatorname{Im}\left(c_{h h v v}\right)$ [34]. The advantage to using (23) is that it circumnavigates the need to unwrap the $h h v v$ phase. Prior to evaluating $d \phi_{h h v v} / d z$ it is practically advantageous to either low-pass filter $R$ and $I$ or use a convolution derivative. In this study we low-pass filtered the data using the inbuilt FIR (Finite Impulse Response) function in MATLAB. In addition to removing high-frequency noise, a rationale for low-pass filtering is that it acts to reduce the effect of $h h v v$ phase excursions due to scattering (refer to previous analysis by [20]). This step therefore enables us to perform a length-scale separation between scattering- and propagationrelated phase behavior, and subsequently isolate the hhvv phase correlation associated with birefringent propagation and smoothly varying, depth-averaged, asymmetry of the COF.

We estimate the error on the evaluation of $d \phi_{h h v v} / d z$ using the following steps. First, we calculate $c_{h h v v}$ for a vertical profile. Second, we use $\left|c_{h h v v}\right|$ and (20) to estimate $\sigma_{\phi_{h h v}}$ as a function of depth. Third, at each depth we generate a statistical ensemble for $c_{h h v v}$ assuming $\phi_{h h v v}$ is a Gaussian random variable. Fourth, we calculate $d \phi_{h h v v} / d z$ for each member of the ensemble using the low-pass filter described above. Fifth, at each depth we estimate the error on the vertical $h h v v$ phase derivative, $\sigma_{d \phi_{h h v} / d z}$, from the standard deviation of the ensemble.

When high angular resolution multi-polarization data is available, the principal axes can be established prior to evaluating fabric asymmetry via comparison with the scattering model predictions. However, in future applications there may be circumstances when it is desirable to estimate fabric asymmetry when there is no prior knowledge if the $h$ and $v$ polarization planes are aligned with the principal axis system (e.g. at an orthogonal cross-over point in a single-polarized airborne survey). Additionally, ground-based multi-polarization data sets may be measured at a coarse angular resolution, again resulting in $h$ and $v$ polarization planes not being aligned with the principal axis system. Appendix $\mathrm{C}$ quantifies the bias in the $E_{2}-E_{1}$ estimate for the case that the measurement polarization planes are not aligned with the principal axes system.

\section{EXPERIMENTAL RESULTS}

The application of the polarimetric coherence method to the MCRDs data set consists of four stages. First, the properties of $\left|c_{h h v v}\right|$ and $\phi_{h h v v}$ are assessed, Sect. V-A. Second, the orientation of the principal axes/fabric eigenvectors are determined via comparison with the scattering model predictions, Sect. V-B. Third, the fabric birefringence is determined using the vertical phase gradient method, Sect. V-C. Fourth, the radarinferred fabric asymmetry is compared with NEEM ice core COF data, Sect. V-D.

\section{A. Depth-azimuth profiles for $c_{h h v v}$}

Figure 5 shows depth-azimuth profiles for $\left|c_{h h v v}\right|, \phi_{h h v v}$, $\operatorname{Re}\left(c_{h h v v}\right)$, and $\operatorname{Im}\left(c_{h h v v}\right)$ at sites $A, B$, and $C$ alongside depth-profiles for the mean (azimuthally averaged) copolarized power, $[P]=10 \log _{10}\left|s_{h h}\right|^{2}$. Plots for the copolarized power anomaly are shown in [14][Fig. 3(a)]. At all 3 sites above an ice depth of $z \sim 1500 \mathrm{~m}\left|c_{h h v v}\right|$ is relatively high and typically ranges from $0.5-0.8$. These shallow/mid depths are associated with greater continuity of $\phi_{h h v v}$ and visible vertical phase gradients are present between $z \sim 800-1500 \mathrm{~m}$. Over depths $z \sim 800-1500 \mathrm{~m}$ the azimuthal symmetry properties in Fig. 5(c)-(e) broadly conform with the matrix model predictions for depth-invariant principal axis in Sect. III. Specifically, as predicted by isotropic and anisotropic scattering models, Fig. 2 and Fig. 3, $\operatorname{Re}\left(c_{h h v v}\right)$ has 90 degree symmetry and $\phi_{h h v v}$ and $\operatorname{Im}\left(c_{h h v v}\right)$ have 180 degree symmetry. At Circle $\mathrm{C}$, however, there is a suggestion of minor counter-clockwise rotation in the principal axis (corresponding to $\sim 5-10^{\circ}$ over ice depths $700-1500 \mathrm{~m}$ ) and this scenario is modelled in Appendix B.

For $z<1500 \mathrm{~m}$ there is a marked decrease in $\left|c_{h h v v}\right|$ which results in a randomization of $\phi_{h h v v}$, and this is also correlated with a step-change reduction in power. This stratigraphic power transition has previously been observed in Greenland radar sounding data and approximately corresponds to the age transition between shallower, more reflective, Holocene ice and deeper, less reflective, Wisconsin ice [35], [36]. At depth $\sim 2500 \mathrm{~m}$ there is an increase in $\left|c_{h h v v}\right|$ associated with the bed reflection. The shallowest ice depth that $c_{h h v v}$ can be evaluated at is $z \sim 400 \mathrm{~m}$ as MCRDs did not record data in shallower ice.

This study does not investigate decorrelation mechanisms for $\left|c_{h h v v}\right|$ and we demonstrate that, for ice depths $z<1500$ $\mathrm{m},\left|c_{h h v v}\right|$ is sufficiently high to infer both fabric orientation and asymmetry. It is, however, apparent that azimuthal periodicity is present for $\left|c_{h h v v}\right|$ with angular minima present, notably at depth $z \sim 1100-1200 \mathrm{~m}$ for Circles A and B. 
(a)
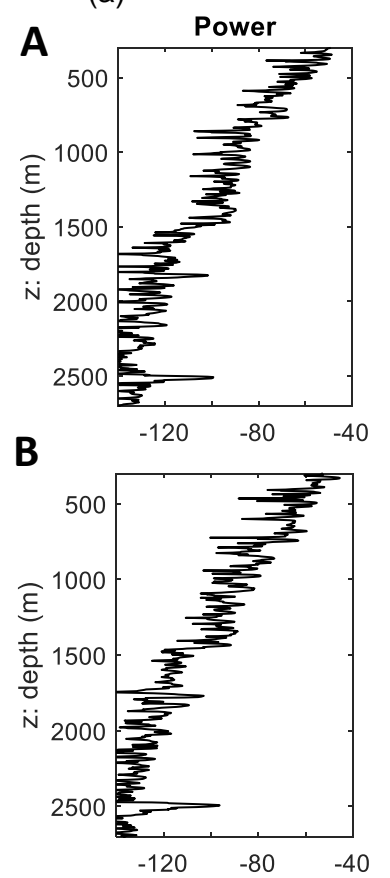

C

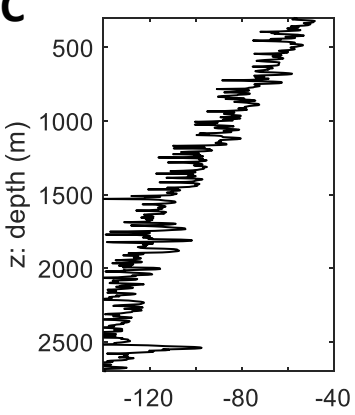

$[P]$ : power $(d B)$ (b)

Coherence magnitude
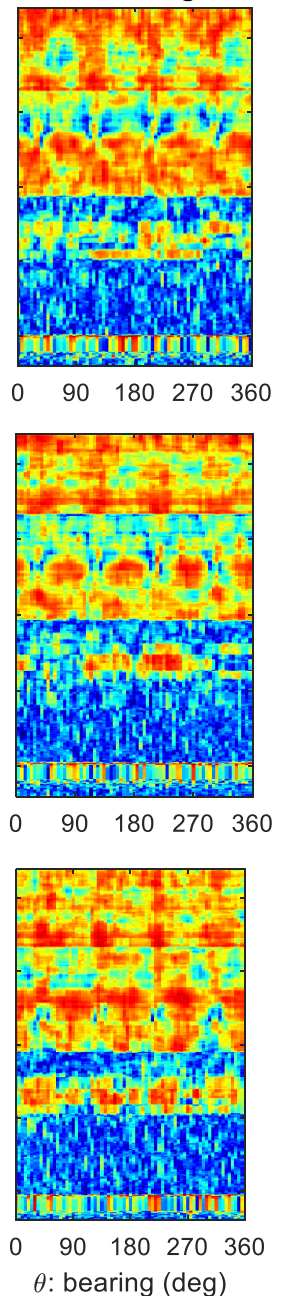

\begin{tabular}{lllll}
\hline & & & & \\
0 & 0.25 & 0.5 & 0.75 & 1
\end{tabular}

$\left|c_{\text {hhvv }}\right|$ (c)
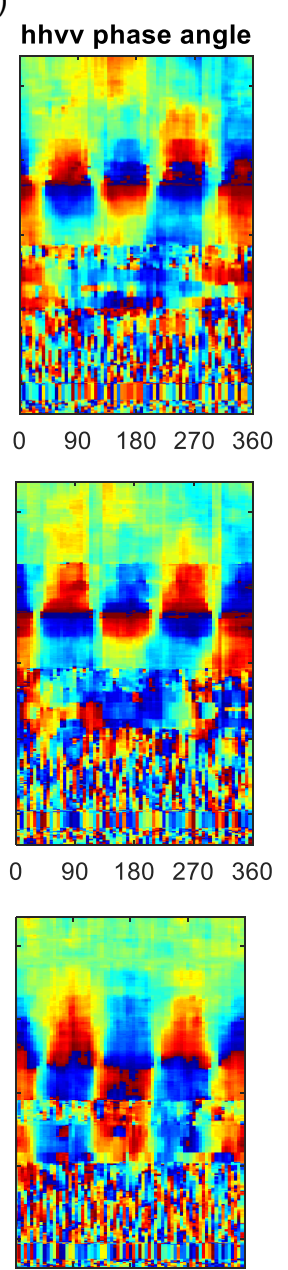

$\begin{array}{lllll}0 & 90 & 180 & 270 & 360\end{array}$ $\theta$ : bearing (deg)

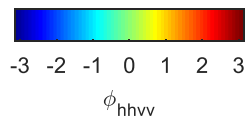

(d)

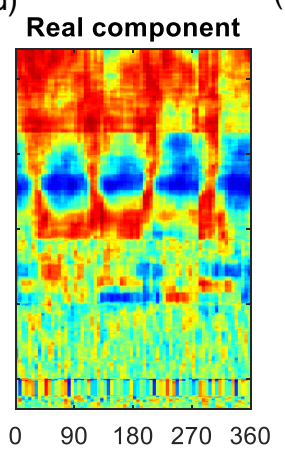

e)
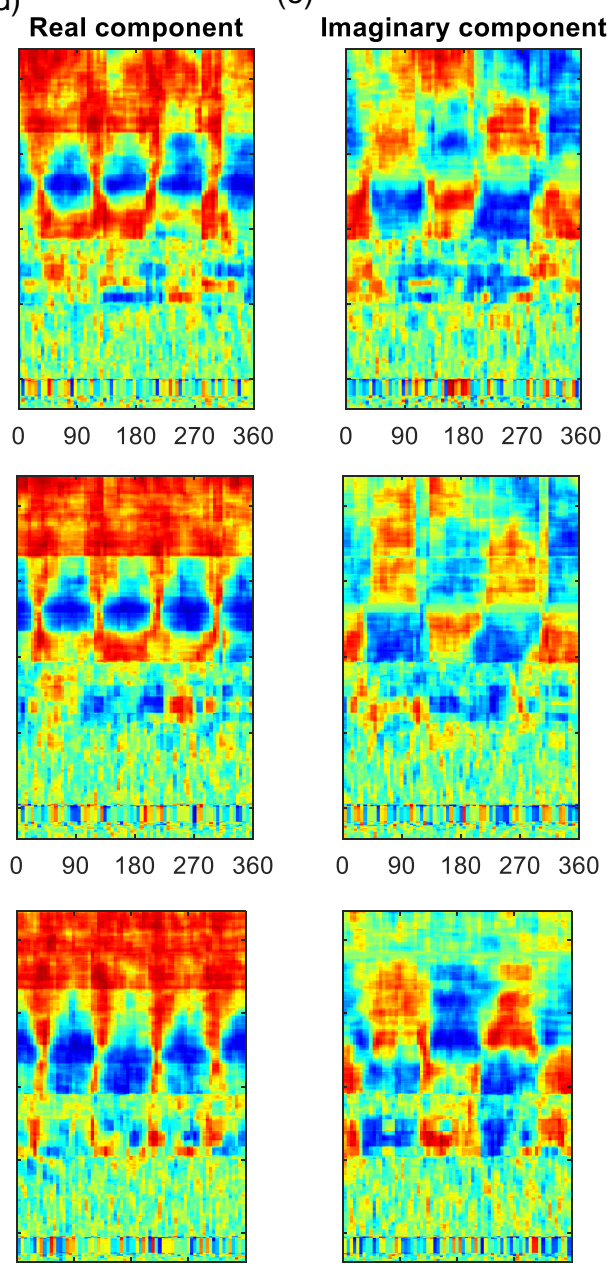

$\begin{array}{lllll}0 & 90 & 180 & 270 & 360\end{array}$

$\theta$ : bearing (deg)

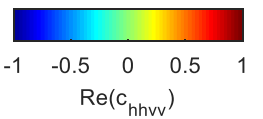

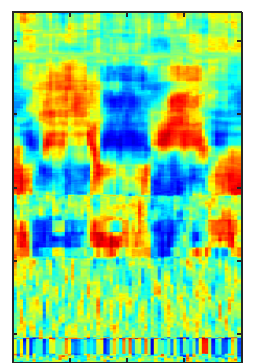

$\begin{array}{lllll}0 & 90 & 180 & 270 & 360\end{array}$

$\theta$ : bearing (deg)

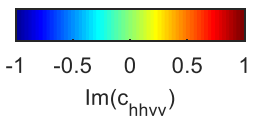

Fig. 5. hhvv coherence, $c_{h h v v}$, as a function of ice depth and bearing angle, $\theta$, for the measurement sites A, B, and C in the NEEM region. (a) Mean (azimuthally averaged) co-polarized power. (b) Coherence magnitude, $\left|c_{h h v v}\right|$. (b) hhvv phase, $\phi_{h h v v}=\arg \left(c_{h h v v}\right)$. (d) $\operatorname{Real}$ component, $\operatorname{Re}\left(c_{h h v v}\right)$. (e) Imaginary component, $\operatorname{Im}\left(c_{h h v v}\right)$.

\section{B. Determination of fabric orientation}

In this study the vertical hhvv phase gradient, $d \phi_{h h v v} / d z$, is used as a diagnostic for fabric orientation. Fig. 6 shows low-passed data from Fig. 5, as used in the evaluation of phase derivative, Sect. IV-C, for the depth range that vertical phase shifting occurs. Fig. 6(b) shows defined azimuthal zones of positive and negative $d \phi_{h h v v} / d z$. Both the isotropic and anisotropic scattering models, (Fig. 2 and Fig. 3), predict that the center angle of these zones corresponds to the principal axes, with $\alpha=0^{\circ}$ corresponding to the center of the positive phase gradient zone and $\alpha=90^{\circ}$ corresponding the center of the negative phase gradient zone. We can therefore infer that the principal axes for sites $A, B$ and $C$ are near-constant with depth, with principal angles $\left(\alpha=0,90^{\circ}\right)$ corresponding to $\theta$ $\approx 75 / 255,165 / 345^{\circ}, \theta \approx 80 / 260,170 / 350^{\circ}$ and $\theta \approx 85 / 265$, $175 / 355^{\circ}$.
The azimuth and phase/depth symmetry comparison between $\operatorname{Re}\left(s_{h h v v}\right)$ and $\operatorname{Im}\left(s_{h h v v}\right)$ (see Fig. 2 and Fig. 3) and $\operatorname{Re}\left(c_{h h v v}\right)$ and $\operatorname{Im}\left(c_{h h v v}\right)$ are generally in very good agreement, and provide an additional visual tool in the modeldata comparison. For example, the minima of $\operatorname{Re}\left(c_{h h v v}\right)$ are located at $\alpha=0,90^{\circ}$ when $\delta=\phi_{h h v v}=\pi$ radians. In the model-data comparison it is, however, important to note that the simulations in Fig. 2 and Fig. 3 are for a fixed birefringence, whereas we later demonstrate that Fig. 5 and Fig. 6 correspond to a birefringence that varies as function of ice depth, (see Sect. V-C and Appendix B for model simulations of depth-varying birefringence).

Comparison between Fig. 6 and Fig. 3 implies the presence of anisotropic scattering. Notably, there are angular zones of oscillating phase gradient close to $\alpha=45,135^{\circ}$ and $\delta=\pi$ radians. 
(a)

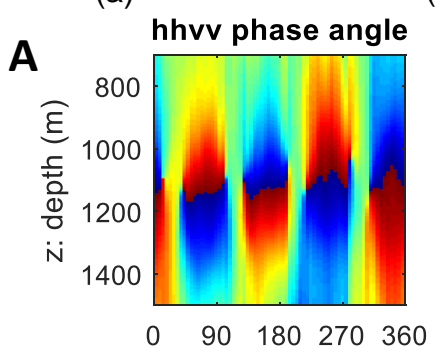

B

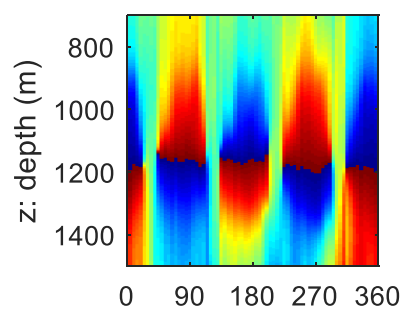

C

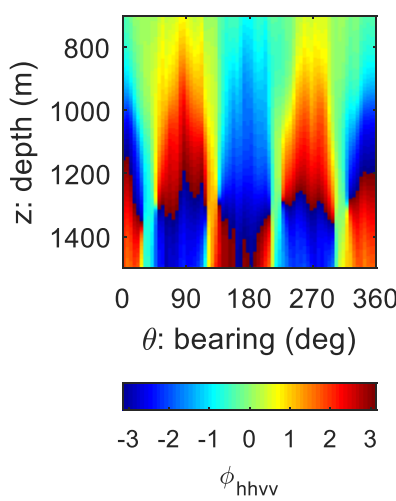

(b)
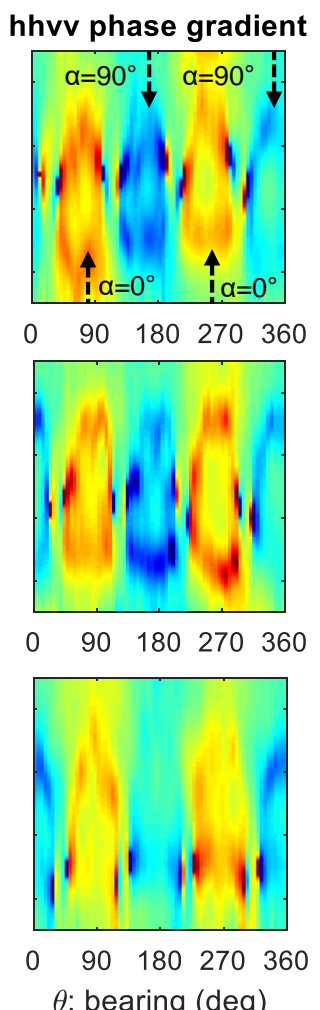

$\theta$ : bearing (deg)

$-0.02-0.01 \quad 0 \quad 0.010 .02$

$\mathrm{d} \phi_{\mathrm{hhvv}} / \mathrm{dz}\left(\mathrm{m}^{-1}\right)$ (c)

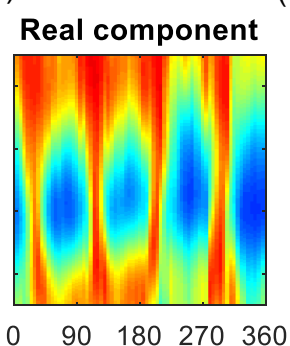

$\begin{array}{lllll}0 & 90 & 180 & 270 & 360\end{array}$
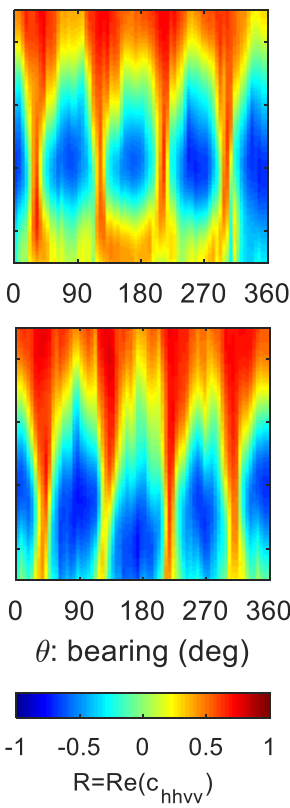

(d)
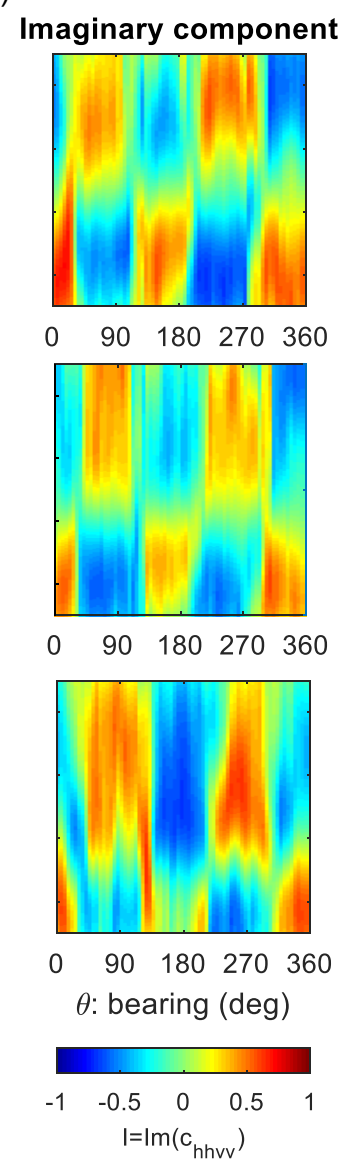

Fig. 6. hhvv coherence phase with low-pass filter applied over mid ice-depths for the measurement sites A, B, and C in the NEEM region. (a) $h h v v$ phase, $\phi_{h h v v}$. (b) $h h v v$ phase gradient, $d \phi_{h h v v} / d z$. (c) Real component, $\operatorname{Re}\left(c_{h h v v}\right)$. (d) Imaginary component, $\operatorname{Im}\left(c_{h h v v}\right)$. $d \phi_{h h v v} / d z$ used as a diagnostic for fabric orientation and an example of the relationship between $\theta$ and the principal axes $\left(\alpha=0,90^{\circ}\right.$ at $\left.\theta \approx 75 / 255,165 / 345^{\circ}\right)$ is indicated in (b) for Circle $\mathrm{A}$, with the principal axis at the center of the azimuthal zones of positive or negative phase gradient. Sites B and C have similar inferred orientation (within 10 degrees of site A).

\section{Determination of birefringence}

Depth profiles for $\left|d \phi_{h h v v} / d z\right|$ and $\Delta \epsilon$ along the inferred principal axes for the three sites are shown in Fig. 7 over the depth range $400-1500 \mathrm{~m}$. The depth profiles were obtained by averaging azimuth bins $\pm 10^{\circ}$ of the inferred values of $\alpha=0^{\circ}$ and $\alpha=90^{\circ}$ given previously in Sect. V-B (i.e. we consider positive and negative gradients together to give a single mean estimate with the implicit assumption that the principal axes are unchanging over the depth range considered). The analysis also considers shallower ice from 400-700 $\mathrm{m}$ and the signs of the $h h v v$ phase gradient along the principal axis are consistent with Fig. 6. We do not consider ice depths $z>1500 \mathrm{~m}$ due to the sharp drop-off in $\left|c_{h h v v}\right|$ in Fig. 5(a).

Values for $\Delta \epsilon$ range from $\sim 0.001-0.006\left(\sim 0.03 \Delta \epsilon^{\prime}-\right.$ $0.18 \Delta \epsilon^{\prime}$ in terms of the single crystal birefringence). Sites $A$ and $B$ both exhibit a marked increase in $\left|d \phi_{h h v v} / d z\right|$ and $\Delta \epsilon$ at $z \sim 800 \mathrm{~m}$. Over depth range $800-1400 \mathrm{~m} \Delta \epsilon$ then weakly oscillates between $\sim 0.003$ and 0.006 , before slightly decreasing for depths $>1400 \mathrm{~m}$. The variation in $\Delta \epsilon$ is less pronounced with ice depth at site $\mathrm{C}$, but the overall form of

\section{Depth profiles for hhvv phase gradient and birefringence}

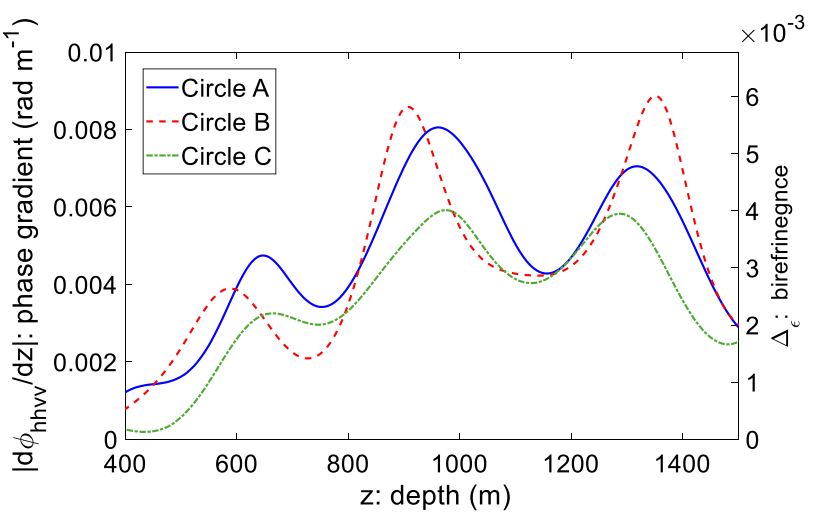

Fig. 7. Depth profiles for the magnitude of the vertical $h h v v$ phase gradient, $\left|d \phi_{h h v v} / d z\right|$, along the principal axes and fabric birefringence, $\Delta \epsilon$.

the relationship is similar to A and B.

Using the approach described in Sect. IV-C we estimated the error in the phase derivative in Fig. 7 to be $\sigma_{d \phi_{h h v v} / d z} \approx$ 
$1 \times 10^{-4} \mathrm{rad} \mathrm{m}^{-1}$ at all three sites. This is an average value across the ice column, but there is only minor depth variation. It corresponds to $\mathrm{a} \sim 2-10 \%$ fractional error, with the greatest fractional error when $\Delta \epsilon$ is lowest.

\section{Determination of COF asymmetry and comparison with NEEM ice core data}

COF measurements from the NEEM ice core were made during 2009-2011 field seasons using an automatic ice texture analyzer [5]. The measurements used the normalized eigenvalue representation described in Sect. II-B and were made at $\mathrm{a} \sim 10 \mathrm{~m}$ vertical resolution from $33 \mathrm{~m}$ down to $2461 \mathrm{~m}$. A depth profile for the orientational eigenvalues is shown [5][Fig. 2] where the eigenvalue convention equivalent to $E_{3}<E_{2}<E_{1}$ is assumed. The NEEM COF depth profiles evolve from being weakly anisotropic in shallow ice to a progressively stronger single maximum at $z \sim 1650 \mathrm{~m}$ that is dated to the Holocene-Wisconsin (HW) climatic transition $(\sim 12,000$ years BP) [5]. At ice depths between $\sim 500$ $1500 \mathrm{~m}$ there is marked splitting between the $E_{2}$ and $E_{1}$ eigenvalues. Approximating the $E_{3}$ eigenvector to be vertical, this is associated with horizontal asymmetry to the COF and a departure from an ideal single maximum fabric/presence of a weak vertical girdle. The NEEM ice core also contains sheath folding at depths $\sim 2200-2400 \mathrm{~m}$ [37], which is associated with fluctuations from a single maximum fabric. However, since these features are at depths where $\left|c_{h h v v}\right|$ is low, Fig. 5(a), we do not discuss them further.

In Fig. 8 the depth profile for $E_{2}-E_{1}$ from the NEEM ice core [5] is compared with radar-inferred profiles derived using (2). The plot includes a low-pass filtered version of $E_{2}-E_{1}$ from the NEEM ice core that matches the windowing method applied to $c_{h h v v}$ when calculating $d \phi_{h h v v} / d z$. The radarinferred $E_{2}-E_{1}$ profiles capture the general (low-frequency) increase that is present in the core data over depths $\sim 400-900$ $\mathrm{m}$, and decrease over depths $\sim 1300-1500 \mathrm{~m}$. Additionally, the radar-inferred $E_{2}-E_{1}$ profiles demonstrate higher frequency (ice-depth wavelength $\sim 200-300 \mathrm{~m}$ ) oscillations in fabric asymmetry that are not present in the ice core data. In particular; all thee circles demonstrate a local minimum in fabric asymmetry at depth $\sim 1150 \mathrm{~m}$. The inferred fabric asymmetry at site $C$ is weaker compared with sites $A$ and $B$, but is still generally consistent with the observed range of $E_{2}-E_{1}$ from the ice core.

A key limitation of the comparison in Fig. 8 is that the $E_{3}$ eigenvector is approximated as vertical in the radar method, and the $E_{2}$ and $E_{1}$ eigenvalues are therefore assumed to represent horizontal $c$-axis concentrations when comparing with the ice core. Appendix A models the effect of a tilt angle upon the radar $E_{2}-E_{1}$ estimate and establishes that a small (worst-case $\sim 20 \%$ ) bias could be present. There are also additional factors which limit the accuracy of the comparison made in Fig. 8. First, the measurement sites are located $\sim$ $5 \mathrm{~km}$ from the core. Second, the ice thickness at the sites can vary by up to $40 \mathrm{~m}$ from the ice core (hence the radiostratigraphy also differs slightly). Third, the ice core data is subject to sources of sample bias. In principle, layer tracing

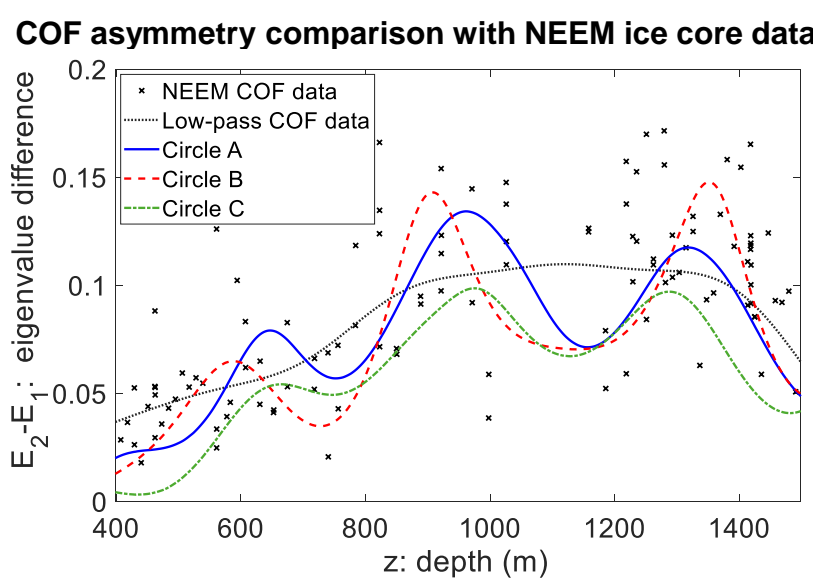

Fig. 8. Comparison of the COF eigenvalue difference, $E_{2}-E_{1}$, between COF data from the NEEM ice core [5] and the radar-inferred values at sites $\mathrm{A}, \mathrm{B}$, and C. $E_{2}-E_{1}$ quantifies the horizontal fabric asymmetry under the assumption that the $E_{3}$ eigenvector is vertical. The effect of a tilt angle upon the comparison is assessed in Appendix A.

(and depth-adjustment) would the enable the second of these factors to be corrected for. However, we do not do this here since there are very small differences in layer depths between the measurement sites and the core (estimated to be $<20 \mathrm{~m}$ over the depth range considered).

Due to rotation of the horizontal ice sections in the drilling processes, the NEEM ice core data cannot directly reveal fabric orientation [9], [10]. We therefore compare our inferred $E_{2}$ eigenvector orientations in Sect. V-B with other radar sounding studies [14], [20] in Sect. VI-B.

\section{DISCUSSION}

\section{A. Interpretation in relation to ice flow in NEEM region}

The radar data analysis in Sect. V-B indicates that the $E_{2}$ eigenvectors (associated with the greatest horizontal $c$-axis concentration) are orientated at bearing angle $\theta \approx 75,80$ and $85^{\circ}$ for sites $A, B$ and $C$ respectively and this result is summarized in Fig. 9. These inferred angles differ slightly from being orientated parallel to the present-day ice divide which is at $\theta \approx 60^{\circ}$ [14]. The results are broadly consistent with a conventional model of ice deformation [3], [7], [9], [13] where the greatest strain component is parallel to the flow/extension direction (perpendicular to the ice divide). In this scenario, since the ice crystal glide plane is perpendicular to the $c$-axis, the COF distribution tends to cluster more along the transverse orientation than along the longitudinal orientation which, in turn, corresponds to the $E_{2}$ eigenvector being orientated parallel to the ice divide. The inferred values of $\theta$ are therefore likely to indicate small departures from this idealized behavior and/or minor regional variation in ice flow direction.

The radar-inferred $E_{2}-E_{1}$ profiles in Fig. 8 are consistent with the COF at NEEM being classified as either a non-ideal single maximum or a weak vertical girdle over ice depths $\sim 800-1500 \mathrm{~m}$. Again, this is supportive of a conventional model of deformation at an ice divide, with weak horizontal asymmetry (associated with lateral tension), present at mid ice 


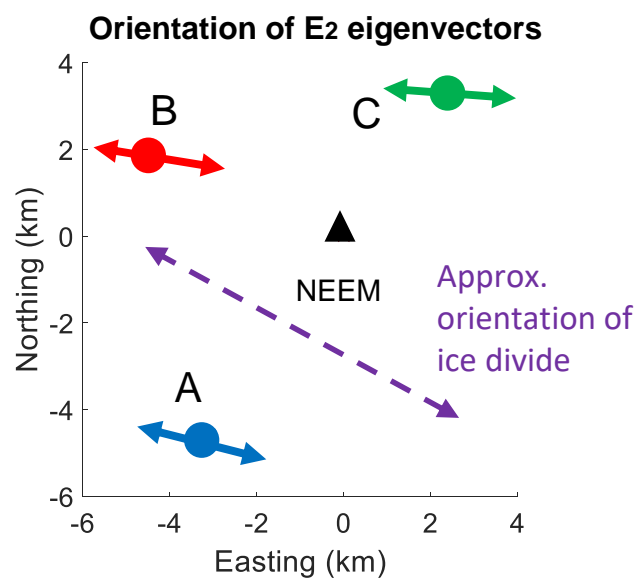

Fig. 9. Schematic showing the inferred orientation of the $E_{2}$ eigenvectors (greatest horizontal $c$-axis concentration under the approximation that the $E_{3}$ eigenvector is vertical) relative to the ice divide.

depths. Site $C$ exhibits weaker fabric asymmetry than sites $A$ and $B$ and likely indicates that local variations in fabric asymmetry can be detected using the method. It is sometimes the case that ice divides undergo flow re-organization which can result in pronounced local variation in the COF [2], [3], [29], [38]. However, the near-constant orientation of the inferred fabric eigenvectors as a function of depth $(z<1500$ $\mathrm{m})$ is consistent with the ice flow direction at each site being orientated in a near-constant direction for the majority of the Holocene period.

\section{B. Comparison with other radar COF studies in NEEM region}

The radar-inferred COF properties in the NEEM region are supported by previous polarimetric coherence analysis of airborne, quad-polarized, POLARIS data (center frequency $435 \mathrm{MHz}$ ) by [20]. In particular, for flight-track orientations parallel and perpendicular to the ice divide (equivalent to $\theta \approx$ $60^{\circ}$ and $\theta \approx 130^{\circ}$ in our study), [20][Fig. 3] observe nearmonotonic positive and negative hhvv phase gradients over ice depths $1000-1500 \mathrm{~m}$. Their orientation result is therefore consistent with the sign of $d \phi_{h h v v} / d z$ at the respective angles in Fig. 6. From a linear approximation of $\left|d \phi_{h h v v} / d z\right|$ over $\sim$ $1000-1500 \mathrm{~m}$ and under the assumption that $E_{3}$ eigenvector is vertical, [20] inferred a COF eigenvalue difference $E_{2}-E_{1} \approx$ 0.12 . Their fabric asymmetry estimate is therefore consistent with both the NEEM COF data and our radar-inferred values in Fig. 8.

Previous analysis of the MCRDs NEEM data set by [14] used the uniaxial single pole model by [11] in conjunction with analysis of polarimetric power anomalies to infer fabric orientation and asymmetry. In their model formulation the $\mathrm{COF}$ has the dielectric properties of a single ice crystal, equivalent to COF eigenvalues $\left(E_{1}, E_{2}, E_{3}\right)=(0,0,1)$ and the horizontal birefringence is given by

$$
\Delta \epsilon=\Delta \epsilon^{\prime} \sin ^{2}(\beta),
$$

where $\beta$ is a tilt angle (see Appendix $\mathrm{A}$ for a derivation as limiting case of the biaxial model). Over depth range 400-1000 $\mathrm{m}$ [14][Fig. 10] inferred that, for the uniaxial model, $\beta$ ranges from $\sim 10-14^{\circ}$. From (24) this results in $\Delta \epsilon \sim 0.0010-0.0020$ and is therefore broadly comparable with Fig. 7 over the respective depth range. [14] also infer a non-linear birefringent phase shift with ice depth (comparable to $\delta$ in this study). Notably Fig. 6 in [14][Fig. 6a] demonstrates an increase in the vertical phase gradient at $\sim 800 \mathrm{~m}$, which is also observed in Fig. 7.

The polarimetric power analysis [14][Fig. 7] assumes that fabric orientation can be determined from polarimetric power differences, which, in turn, are related to anisotropic scattering coefficients. However, in this study we assume fabric orientation can be determined via a birefringent propagation model based upon a local average of the COF asymmetry in the horizontal plane. From their approach, [14] conclude that the greatest horizontal $c$-axis concentration at NEEM is orientated near-perpendicular to the ice divide in shallower ice $(\sim 450$ $1065 \mathrm{~m})$ and near-parallel in deeper ice ( 1375 to $1575 \mathrm{~m})$. Their inferred fabric orientation is therefore not consistent with this study in shallower ice, but is consistent in deeper ice.

\section{Comparison between matrix model formulation for polari- metric coherence and power anomaly methods}

The polarimetric coherence method in this study and the power anomaly method by [2], [13] are both based upon the same matrix backscatter model and therefore make similar assumptions about the fabric orientation tensor, Sect. II-B, and the model physics, Sect. III. The coherence method focuses on radar phase to infer COF properties which has some advantages and limitations, and we now compare the two methods for the case of co-polarized measurements.

The first advantage of the coherence method is that it removes ambiguity regarding the inferred dielectric principal axes. Specifically, for the case of birefringent propagation with isotropic scattering co-polarized power measurements are predicted to have 90 degree azimuthal symmetry with power minima at $\alpha=45,135^{\circ}$ and maxima at $\alpha=0,90$ - (aligned with the principal axes) [2][Fig. 7(a)], [13][Fig. 5(a)]. Using power anomalies to infer orientation, there is therefore ambiguity regarding whether the $h$ or $v$ polarization plane is aligned with the higher or lower principal permittivity (i.e. the $E_{2}$ and $E_{1}$ eigenvectors). However, due to the 180 degree periodicity of $\phi_{h h v v}$ in Fig. 2 - in particular the sign of $d \phi_{h h v v} / d z$ - the coherence method provides a robust way to infer the $E_{2}$ and $E_{1}$ eigenvectors. The second advantage of the coherence method is that the symmetry properties of $\phi_{h h v v}$ are robust to the inclusion of anisotropic scattering (see Fig. 3 in this study, [2][Fig. 7(c,d)], [13][Fig. 5])). Notably, the sign of the $d \phi_{h h v v} / d z$ along the principal axes is preserved and the principal axes remain aligned with the azimuthal center of the zones of positive and negative $d \phi_{h h v v} / d z$. By comparison, in co-polarized power analysis the inclusion of anisotropic scattering (which in isolation has 180 degree periodicity) acts to break the 90 degree azimuthal periodicity of birefringent propagation. The third advantage of the coherence method is that it enables the depth-evolution of birefringence and fabric asymmetry to be estimated (nominal resolution $\sim 100 \mathrm{~m}$ in this 
study). Using power anomalies, depth-averaged birefringence is assessed at a coarser resolution over depth intervals of $\delta=$ $\pi$ radians (established from the presence of the co-polarized nodes at $\alpha=45,135^{\circ}$ ) [13].

A clear limitation of the coherence method is that, due to the depth-averaging when calculating $c_{h h v v}$, it is not suited to detect rapid fabric transitions (e.g. [28], [29]). Analysis of polarimetric power anomalies are better suited for this task since the polarimetric effects of anisotropic scattering for a particular reflector can be better isolated. Additionally, phasecoherent radar data is a pre-requisite for the coherence method whereas it is not required for the power anomaly method.

\section{Future applications of the coherence method}

In general quad-polarized radar sounding data is relatively rare, being confined to smaller airborne surveys or ground campaigns. Subsequently, with the rationale that future applications of the coherence method could be applied to singlepolarized radar surveys, we used varying track-orientation of the single-polarized MCRDs data set to co-register copolarized ( $h h$ and $v v$ ) measurements.

In addition to ground-based 'turning circle' data, an orthogonal cross-over point in a ground or airborne survey grid also produces a $h h-v v$ measurement pair. In this scenario, however, the $h$ and $v$ polarization planes will not necessarily be aligned with the dielectric principal axes/fabric eigenvectors. The angular bias estimation for $\left|d \phi_{h h v v} / d z\right|$ (Appendix C) demonstrates that even fairly large departures from the principal axes can produce useful estimates of fabric asymmetry (e.g. at $\sim 20$ degrees away from the principal axes there is a maximum $\sim$ $\pm 20 \%$ bias). Additionally, the sign of $d \phi_{h h v v} / d z$ can be used to place approximate constraints upon the orientation of the principal axes. However, accurately resolving the orientation of the principal axes from a single $h h-v v$ pair is likely to be difficult task. Therefore, when applied to cross-over points, the coherence method is likely to be most useful in a hypothesis validation framework: for example, testing for evidence of flow re-organization or fabric strengthening associated with dynamical processes.

\section{SUMMARY AND CONCLUSIONS}

In this study we developed a polarimetric coherence (phasebased) radar sounding method to determine the COF of polar ice-sheets. We used a polarimetric matrix backscatter model to simulate the phase of the (deterministic) hhvv cross-term, $s_{h h v v}$, which was then related to the phase of the data-derived (stochastic) hhvv coherence, $c_{h h v v}$. This model-data comparison enabled us to demonstrate that, for multi-polarization plane data, the azimuth and depth-dependence of the vertical gradient of the $h h v v$ coherence phase, $d \phi_{h h v v} / d z$, is sufficient to unambiguously determine the dielectric principal axes and the birefringence in the horizontal plane (under the assumption that one of the principal axes is vertical). These dielectric properties were then related to the eigenvectors of the fabric orientation tensor and the difference between horizontal eigenvalues (a measure of the degree of horizontal fabric asymmetry).
The method was demonstrated using ground-based 'turning circle' measurements from the MCRDs radar system at three sites surrounding the NEEM ice core, Greenland. At all three sites, the $E_{2}$ eigenvector (greatest horizontal $c$-axis concentration) was inferred to be close to parallel to the ice divide (within $\sim 25$ degrees) and near-unchanging with ice-depth $(z$ $<1500 \mathrm{~m}$ ), which is consistent with previous analysis of data from the airborne POLARIS radar system [20]. The orientation results are also consistent with both a conventional model of ice deformation at an ice divide where a lateral tension component is present [9], [13] and a stable flow orientation in the NEEM region throughout the Holocene period. We then validated the radar-inferred horizontal eigenvalue difference with COF data from the NEEM ice core [5], and demonstrated that the method can determine the depth profile of the $E_{2}-E_{1}$ eigenvalue difference (horizontal fabric asymmetry).

A practical advantage to the coherence method is that $\mathrm{COF}$ properties can be obtained purely from single/co-polarized measurements with track/antenna orientation being used to coregister $h h$ and $v v$ polarization pairs. Due to the statistical averaging of $h h v v$ coherence, the method is tuned to determine (locally) depth-averaged properties of the COF associated with birefringent propagation (vertical length scale $\sim 100 \mathrm{~m}$ in this study). Importantly, under the assumption that the principal axes of propagation and scattering layers are aligned, the hhvv phase has preserved symmetry properties between isotropic and anisotropic scattering models. This implies that the method can be extended with confidence to different fabric regimes across the polar ice sheets.

\section{APPENDIX A}

ASSESSMENT OF THE EFFECT OF A TILT ANGLE UPON THE FABRIC ASYMMETRY ESTIMATE

When the $E_{3}$ eigenvector is not aligned with the vertical (i.e. there is a tilt angle present) there is an additional source of horizontal birefringence [30]. In order to assess the effect of this upon the $E_{2}-E_{1}$ (fabric asymmetry) estimate we consider rotating the principal dielectric tensor, (1). In this analysis we fix the $(x, y, z)$ coordinate system, previously coincident with the fabric eigenvectors $(1,2,3)$, to continue to represent a horizontal ice-sheet coordinate system.

We consider two different rotation scenarios that provide a means to assess end-member behavior. The first scenario considers a rotation of the $(1,3)$ axes in the $(x, z)$ plane, and the second considers a rotation of the $(2,3)$ axes in the $(y, z)$ plane, Fig. 10. The rotation transformations between the $(1,2,3)$ and the $(x, y, z)$ system are formulated for tilt angle $\beta$, which represents the angle between the $E_{3}$ eigenvector and the vertical, and are of the form $R(\beta) \underline{\underline{\epsilon}} R^{\prime}(\beta)$ where $R(\beta)$ represents a $2 \mathrm{D}$ rotation matrix as in (8). As they are in the plane of the principal axes, these rotation transformations preserve the diagonalized form of the horizontal part of dielectric tensor, with the principal permittivity in the plane of rotation replaced with a horizontal (effective) permittivity. Consequently, polarization mode-separation still occurs for polarization planes aligned with the $(x, y)$ coordinates and the propagation matrices in the scattering model, (9), still apply. 
For rotation in the $(x, z)$ plane the horizontal permittivities are given by:

$$
\begin{aligned}
& \epsilon_{x}=\left(\epsilon_{\perp c}+E_{1} \Delta \epsilon^{\prime}\right) \cos ^{2}(\beta)+\left(\epsilon_{\perp c}+E_{3} \Delta \epsilon^{\prime}\right) \sin ^{2}(\beta), \\
& \epsilon_{y}=\epsilon_{\perp c}+E_{2} \Delta \epsilon^{\prime},
\end{aligned}
$$

and for rotation in the $(y, z)$ plane the horizontal permittivities are given by:

$$
\begin{aligned}
& \epsilon_{x}=\epsilon_{\perp c}+E_{1} \Delta \epsilon^{\prime}, \\
& \epsilon_{y}=\left(\epsilon_{\perp c}+E_{2} \Delta \epsilon^{\prime}\right) \cos ^{2}(\beta)+\left(\epsilon_{\perp c}+E_{3} \Delta \epsilon^{\prime}\right) \sin ^{2}(\beta) .
\end{aligned}
$$

It follows from the previous definition, (2), that the horizontal birefringence is

$$
\Delta \epsilon=\Delta \epsilon^{\prime}\left|\left(E_{2}-E_{1}\right)-\sin ^{2}(\beta)\left(E_{3}-E_{1}\right)\right|,
$$

for rotation in the $(x, z)$ plane, and

$$
\Delta \epsilon=\Delta \epsilon^{\prime}\left|\left(E_{2}-E_{1}\right)+\sin ^{2}(\beta)\left(E_{3}-E_{2}\right)\right|,
$$

for rotation in the $(y, z)$ plane. (The modulus of the eigenvalue differences are now considered so that $\Delta \epsilon>0$ holds for all values of $\left.E_{1}, E_{2}, E_{3}\right)$. For a uniaxial model [11], [14], with COF eigenvalues $\left(E_{1}, E_{2}, E_{3}\right)=(0,0,1)$, (27) and (28) both reduce to $\Delta \epsilon=\Delta \epsilon^{\prime} \sin ^{2}(\beta)$.

Field measurements for $\beta$ are estimated in relation to the core axis, are summarized for different ice core sites by [30], and range from $3-10^{\circ}$. At NEEM a mean value of $\beta=9.6^{\circ}$ over the depth range $350-1000 \mathrm{~m}$ was measured by [14]. To assess potential biases in the estimation of $E_{2}-E_{1}$ for assuming $\beta$ $=0$ when $\beta>0$ we consider the ratios

$$
\frac{\left(E_{2}-E_{1}\right)_{\beta=0}}{\left(E_{2}-E_{1}\right)_{\beta>0}}=1-\sin ^{2}(\beta) \frac{\left(E_{3}-E_{1}\right)_{\beta>0}}{\left(E_{2}-E_{1}\right)_{\beta>0}},
$$

for $(x, z)$ rotation and

$$
\frac{\left(E_{2}-E_{1}\right)_{\beta=0}}{\left(E_{2}-E_{1}\right)_{\beta>0}}=1+\sin ^{2}(\beta) \frac{\left(E_{3}-E_{2}\right)_{\beta>0}}{\left(E_{2}-E_{1}\right)_{\beta>0}},
$$

for $(y, z)$ rotation where the $\beta=0$ subscripts notate eigenvalue differences for no tilt angle (as previously inferred in the data analysis in Fig. 8) and $\beta>0$ subscripts notate eigenvalue differences for non-zero tilt angle (as modelled here). Equations (29) and (30) are derived by equating (27) and (28) with (2). They represent the fractional estimation bias in $E_{2}-E_{1}$, with $E_{2}-E_{1}>1$ representing an overestimation in the prior data analysis and $E_{2}-E_{1}<1$ representing an underestimation.

To quantify the potential estimation bias at NEEM, we evaluated (29) and (30) for ice fabrics with eigenvalues $\left(E_{1}, E_{2}, E_{3}\right)_{\beta>0}=(0.20,0.25,0.55)$ and $\left(E_{1}, E_{2}, E_{3}\right)_{\beta>0}=$ $(0.10,0.20,0.70)$ which is broadly representative of ice fabric at $z \sim 500 \mathrm{~m}$ and $z \sim 1000 \mathrm{~m}$ in the NEEM ice core [5][Fig. 2]. Results for $0 \leq \beta \leq 10^{\circ}$ are shown in Fig. 10, and show that rotation in the $(x, z)$ plane results in negative bias $\left(E_{2}-E_{1}\right.$ is underestimated by assuming $\beta=0^{\circ}$ in the data analysis) and rotation in the $(y, z)$ plane results in positive bias $\left(E_{2}-E_{1}\right.$ is overestimated by assuming $\beta=0^{\circ}$ ). Fig. 10 also shows that a tilt angle $\beta=10^{\circ}$ (comparable to tilt angles measured at NEEM by [14]) results biases to the $E_{2}-E_{1}$ estimate $\sim \pm$ $20 \%$. These biases are of comparable size to the variation in the $E_{2}-E_{1}$ estimate between measurement sites in Fig. 8 and therefore do not impact significantly upon the comparison that is made.

\section{Bias in COF asymmetry estimate due to tilt angle}

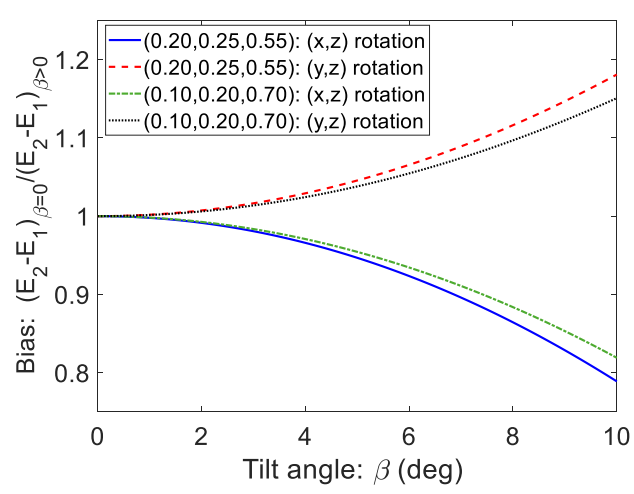

Z

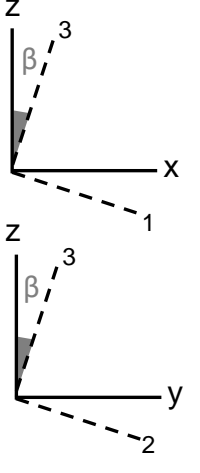

Fig. 10. Bias in $E_{2}-E_{1}$ estimate due to presence of tilt angle, $\beta$, for rotation in the $(x, z)$ and $(y, z)$ planes. Two different ice fabrics are modelled with eigenvalues $\left(E_{1}, E_{2}, E_{3}\right)_{\beta>0}=(0.20,0.25,0.55)$ and $\left(E_{1}, E_{2}, E_{3}\right)_{\beta>0}=$ $(0.10,0.20,0.70)$. A bias $>1$ indicates overestimation of $E_{2}-E_{1}$ due to non-zero $\beta$ and a bias $<1$ indicates underestimation.

\section{APPENDIX B}

\section{EXTENSIONS TO THE SCATTERING MODEL SIMULATIONS:} DEPTH-VARYING BIREFRINGENCE AND ORIENTATION

We now extend the scattering model, Sect. III, to consider the behavior of $s_{h h v v}$ for two scenarios: first, depth-varying fabric birefringence/asymmetry; second, depth-varying fabric orientation. The scattering matrix model equations for depthvarying birefringence are the same as in Sect. III-B, and the difference from the constant birefringence case arises from (12), the relationship between the phase shift and the birefringence. If, for illustrative purposes, a linearly increasing birefringence with depth is a now assumed, $\Delta \epsilon=\rho\left(z-z_{0}\right)$ where $\rho$ is a constant, then (12) becomes

$$
\delta=\frac{4 \pi f}{c} \frac{\rho}{2 \sqrt{\bar{\epsilon}}}\left(z-z_{0}\right)^{2}+\delta_{0}
$$

and there is a quadratic relationship between $\delta$ and $z$. The top row of Fig 11 shows results for $\rho=3.54 \times 10^{-5} \mathrm{~m}^{-1}$ (a value chosen so that $\delta=2 \pi$ occurs at $z=1000 \mathrm{~m}$ ) and isotropic scattering layers.

To model depth-varying fabric orientation we use a more general form of the scattering model (see [13][eqs. (9)-(12)]) that incorporates differing azimuthal angles for different layers of the ice sheet. We consider a linearly increasing counterclockwise rotation of the fabric eigenvectors with ice depth. Computationally, this is achieved by setting $\alpha \rightarrow \alpha+\nu\left(z-z_{0}\right)$ where $\nu$ is a constant and $\nu\left(z-z_{0}\right)$ is the angular offset of the principal axis of the scattering layer (depth $z$ ) from the initial ice depth (depth $z_{0}$ ). Results for $\nu=0.0125^{\circ} \mathrm{m}^{-1}$ (corresponding to an angular offset of $22.5^{\circ}$ at $1000 \mathrm{~m}$ ) are shown on the bottom row of Fig. 11 for isotropic scattering. It is evident that there is a rotation of the ' 90 degree zones' of positive and negative gradients for $d \phi_{h h v v} / d z$ a function of ice depth and the center angle of these zones is shown in Fig. 11(b). 
Depth-varying birefringence
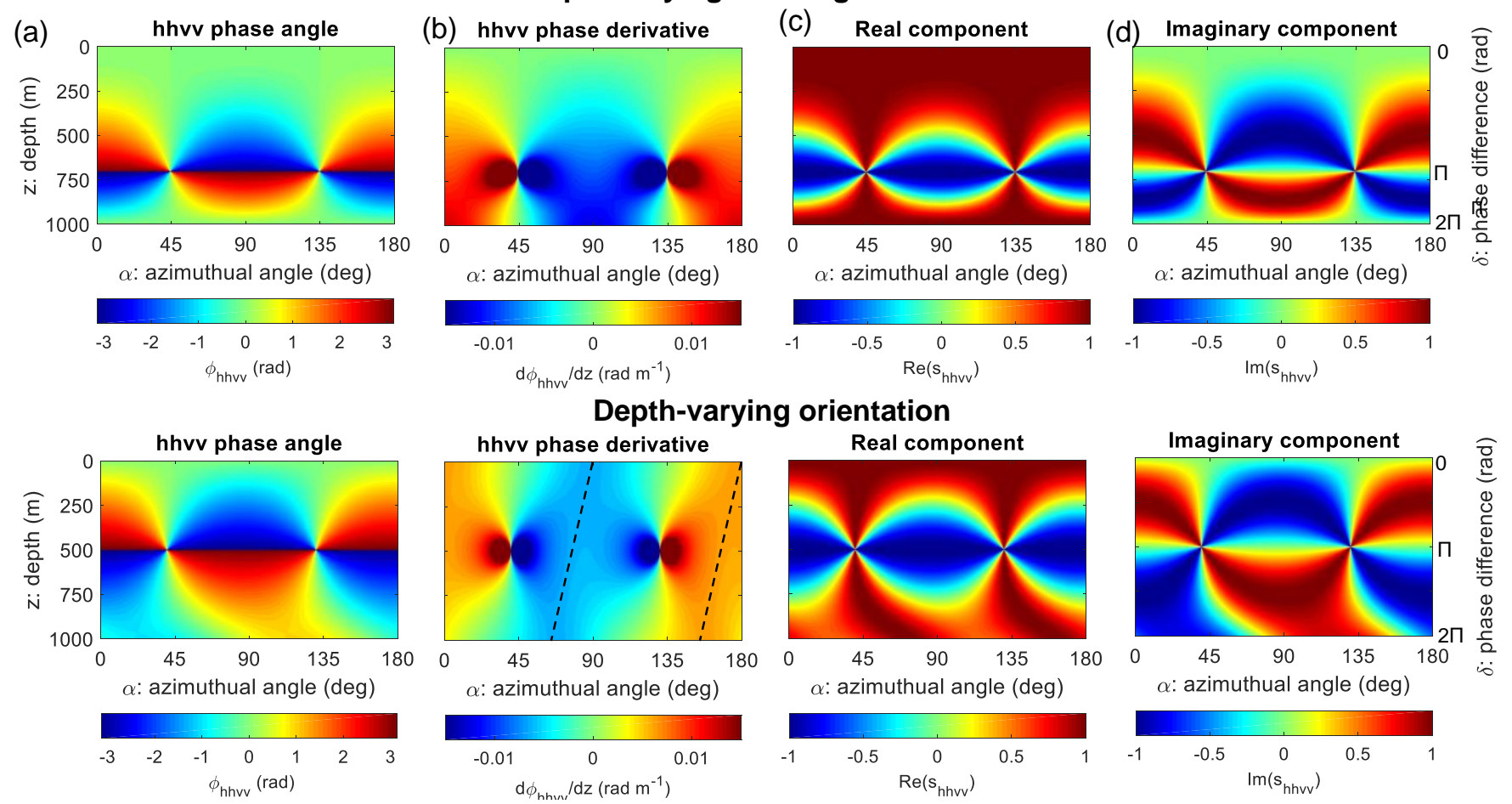

Fig. 11. Matrix backscatter model results for depth-varying birefringence (top row) and depth-vaying orienation (bottow row). (a) $h h v v$ phase, $\phi_{h h v v}=$ $\arg \left(s_{h h v v}\right)$. (b) Vertical $h h v v$ phase gradient, $d \phi_{h h v v} / d z$. (c) Real component, $\operatorname{Re}\left(s_{h h v v}\right)$, (d) Imaginary component, $\operatorname{Im}\left(s_{h h v v}\right)$. The azimuthal angle in the bottom row is referenced to $z=0$ and the rotation of the principal axes are indicated by the dashed black line in plot (b).

\section{APPENDIX C}

ASSESSMENT OF USING A NON-PRINCIPAL AZIMUTHAL ANGLE FOR THE FABRIC ASYMMETRY ESTIMATE

We now consider estimating $E_{2}-E_{1}$ (fabric asymmetry) for the general case when the $h$ and $v$ polarization planes are not aligned with the principal axes (i.e. the hhvv phase derivative, (22), is evaluated for $\alpha \neq 0,90^{\circ}$ ). This investigation is motivated by future applications of the coherence method for ground-surveys where there is a coarse angular resolution or at orthogonal cross-over points in survey grids. In general, $d \phi_{h h v v} / d z$ is a function of both $\alpha$ and $\delta$ (see Fig. 2 and Fig. 3 ). For fixed $\alpha$, and using the chain rule, it follows that

$$
\left|\frac{d \phi_{h h v v}}{d z}\right|=\frac{d \delta}{d z}\left|\frac{d \phi_{h h v v}}{d \delta}\right| \text {. }
$$

When $\alpha=0,90^{\circ},\left|d \phi_{h h v v} / d \delta\right|=1$ and (32) reduces to (21). We can quantify the effect that non-principal $\alpha$ has upon the $E_{2}-E_{1}$ estimate by equating (22) with (32). Following a similar approach to Appendix A, we can then express the estimation bias as the ratio

$$
\frac{\left(E_{2}-E_{1}\right)_{\alpha=0}}{\left(E_{2}-E_{1}\right)_{\left|\alpha-\alpha_{0}\right|>0}}=\left|\frac{d \phi_{h h v v}}{d \delta}\right|
$$

where $\left|\alpha-\alpha_{0}\right|$ with $\alpha_{0}=0,90^{\circ}$ quantifies the angular distance from the principal angles. As for (29) and (30), ratios $>1$ correspond to overestimation of $E_{2}-E_{1}$ in the data analysis and $<1$ correspond to underestimation. Sections of $\left|d \phi_{h h v v} / d \delta\right|$ for constant $\left|\alpha-\alpha_{0}\right|$ are shown in Fig. 12 for isotropic scattering. For $\left|\alpha-\alpha_{0}\right|=20^{\circ}$ (approximately the coarsest angular resolution that is likely to occur in a ground survey) the maximum bias is $\sim \pm 20 \%$.

\section{Bias in COF asymmetry estimate due to non-principal angle}

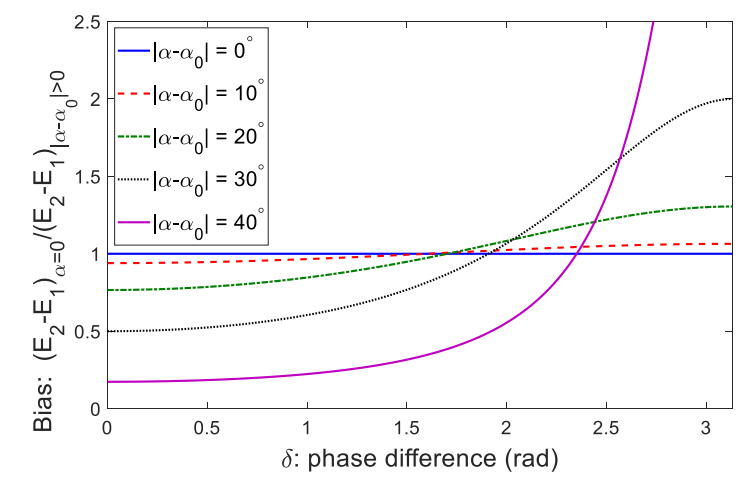

Fig. 12. Bias in $E_{2}-E_{1}$ (fabric asymmetry) estimate when measurement system is not aligned with principal axis system. A bias $>1$ indicates an overestimation of $E_{2}-E_{1}$ due to evaluation at a non-principal angle and a bias $<1$ indicates an underestimation.

\section{ACKNOWLEDGMENTS}

TMJ would like to acknowledge support from EU Horizons 2020 grant 747336-BRISRES-H2020-MSCA-IF-2016. We would like to thank Reinhard Drews, University of Tubingen, and two anonymous reviewers for their constructive comments that improved this study. We would like to thank 
Jonathan Bamber, University of Bristol, and Maurine Montagnat, Univ. Grenoble Alpes, for their comments.

\section{REFERENCES}

[1] R. B. Alley, "Fabrics in polar ice sheets: Development and prediction," Science, vol. 240, no. 4851, pp. 493-495, 1988.

[2] K. Matsuoka, D. Power, S. Fujita, and C. F. Raymond, "Rapid development of anisotropic ice-crystal-alignment fabrics inferred from englacial radar polarimetry, central West Antarctica," Journal of Geophysical Research: Earth Surface, vol. 117, no. 3, pp. 1-16, 2012.

[3] A. M. Brisbourne, C. Martin, A. M. Smith, A. F. Baird, J. M. Kendall, and J. Kingslake, "Constraining Recent Ice Flow History at Korff Ice Rise, West Antarctica, Using Radar and Seismic Measurements of Ice Fabric," Journal of Geophysical Research: Earth Surface, vol. 124, pp. 175-194-373, 2019.

[4] J. H. Kennedy, E. C. Pettit, and C. L. D. I. Prinzio, "The evolution of crystal fabric in ice sheets and its link to climate history," Journal of Glaciology, vol. 59, no. 214, pp. 357-373, 2013.

[5] M. Montagnat, N. Azuma, J. Eichler, S. Fujita, S. Kipfstuhl, and D. Samyn, "Fabric along the NEEM ice core, Greenland, and its comparison with GRIP and NGRIP ice cores," The Cryosphere, vol. 8, pp. 1129-1138, 2014

[6] P. V. Hobbs, Ice Physics. Clarendon, Oxford, 1974, pp. 274-280.

[7] N. Azuma, "A flow law for anisotropic ice and its application to ice sheets," Earth and Planetary Science Letters, vol. 128, pp. 601-614, 1994.

[8] Y. Ma, O. Gagliardini, C. Ritz, F. Gillet-chaulet, and M. Montagnat, "Enhancement factors for grounded ice and ice shelves inferred from an anisotropic ice-flow model," Journal of Glaciology, vol. 56, no. 199, pp. 805-812, 2010.

[9] Y. Wang, T. Thorsteinsson, J. Kipfstuhl, H. Miller, D. Dahl-Jensen, and H. Shoji, "A vertical girdle fabric in the NorthGRIP deep ice core," Journal of Glaciology, vol. 35, pp. 515-520, 2002.

[10] H. Faria, J. Freitag, and S. Kipfstuhl, "Polar ice structure and the integrity of ice-core paleoclimate records," Quaternary Science Reviews, vol. 29 , pp. 338-351, 2010.

[11] N. D. Hargreaves, "The polarization of radio signals in the radio echo sounding of ice sheets," Journal of Physics D: Applied Physics, vol. 10, pp. 1285-1304, 1977.

[12] - "The radio-frequency birefringence of polar ice," Journal of Glaciology, vol. 21, no. 85, pp. 301-313, 1978.

[13] S. Fujita, H. Maeno, and K. Matsuoka, "Radio-wave depolarization and scattering within ice sheets: a matrix-based model to link radar and icecore measurements and its application," Journal of Glaciology, vol. 52, no. 178, pp. 407-424, 2006.

[14] J. Li, J. A. V. González, C. Leuschen, A. Harish, P. Gogineni, M. Montagnat, I. Weikusat, F. Rodriguez-morales, and J. Paden, "Multi-channel and multi-polarization radar measurements around the NEEM site," The Cryosphere, vol. 12, pp. 2689-2705, 2018.

[15] K. Matsuoka, T. Furukawa, S. Fujita, H. Maeno, S. Uratsuka, R. Naruse, and O. Watanabe, "Crystal orientation fabrics within the Antarctic ice sheet revealed by a multipolarization plane and dual-frequency radar survey," Journal of Geophysical Research, vol. 108(B10), pp. 1-17, 2003.

[16] C. S. M. Doake, H. F. J. Corr, A. Jenkins, K. W. Nicholls, and C. Stewart, "Interpretation of polarization behaviour of radar waves transmitted through antarctic ice shelves," Proceedings of the Workshop on POLinSAR - Applications of SAR Polarimetry and Polarimetric Interferometry (ESA SP-529), 2003.

[17] S. R. Cloude and K. P. Papathanassiou, "Polarimetric SAR interferometry," IEEE Transactions on Geoscience and Remote Sensing, vol. 36, no. 5, pp. 1551-1565, Sept 1998.

[18] A. Moreira, P. Prats-iraola, M. Younis, G. Krieger, I. Hajnsek, and K. P. Papathanassiou, "A tutorial on synthetic aperture radar," IEEE Geoscience and Remote Sensing Magazine, no. 1, pp. 14-16, 2013.

[19] J. Dall, "Polarimetric ice sounding at p-band: First results," IEEE 2009 International Geoscience and Remote Sensing Symposium (IGARSS 2009), Cape Town, July 2009, pp. 1024-1027, 2009.

[20] _ - "Ice sheet anisotropy measured with polarimetric ice sounding radar," 30th International Geoscience and Remote Sensing Symposium (IGARSS 2010), 2530 July 2010, Honolulu, HI, USA., pp. 2507-2510, 2010.

[21] J. Dall, S. S. Kristensen, V. Herna, C. C. Krozer, J. Kusk, A. Vidkjær, J. Skou, N. Balling, and E. L. Christensen, "ESA's POLarimetric Airborne Radar Ice Sounder (POLARIS): design and first results," IET Radar, Sonar and Navigation, vol. 4, no. 3, pp. 488-496, 2009.
[22] J. L. Vazquez-Roy, V. Krozer, and J. Dall, "Wideband dual-polarization microstrip patch antenna array for airborne ice sounder," IEEE Antennas and Propagation Magazine, vol. 54, no. 4, pp. 98-107, 2012.

[23] C. S. M. Doake, H. F. J. Corr, and A. Jenkins, "Polarization of radio waves transmitted through Antarctic ice shelves," Annals of Glaciology, vol. 34, pp. 165-170, 2002.

[24] S. Fujita, T. Matsuoka, T. Ishida, K. Matsuoka, and S. Mae, "A summary of the complex dielectric permittivity of ice in the megahertz range and its applications for radar sounding of polar ice sheets," Physics of Ice Core Records, vol. 104, pp. 185-212, 2000.

[25] T. Matsuoka, S. Fujita, and S. Mae, "Effect of temperature on dielectric properties of ice in the range 5-39 ghz," Journal of Applied Physics, vol. 80, no. 109, 1996.

[26] N. Woodcock, "Specification of fabric shapes using an eigenvalue method," Geological Society of America Bulletin, vol. 88, pp. 1231$1236,1977$.

[27] A. Maurel, F. Lund, and M. Montagnat, "Propagation of elastic waves through textured polycrystals: application to ice," Proc. R. Soc. A, 2015.

[28] O. Eisen, I. Hamann, S. Kipfstuhl, D. Steinhage, and F. Wilhelms, “ Direct evidence for continuous radar reflector originating from changes in crystal-orientation fabric," The Cryosphere, vol. 1, pp. 1-10, 2007.

[29] R. Drews, O. Eisen, D. Steinhage, I. Weikusat, S. Kipfstuhl, and F. Wilhelms, "Potential mechanisms for anisotropy in ice-penetrating radar data," Journal of Glaciology, vol. 58, no. 209, pp. 613-624, 2012.

[30] K. Matsuoka, L. Wilen, S. Hurley, and C. Raymond, "Effects of Birefringence Within Ice Sheets on Obliquely Propagating Radio Waves," IEEE Transactions on Geoscience and Remote Sensing, vol. 47, no. 5, pp. 1429-1443, may 2009.

[31] S. Fujita, H. Maeno, S. Uratsuka, T. Furukawa, S. Mae, Y. Fujii, and O. Watanabe, "Nature of radio echo layering in the Antarctic ice sheet detected by a two-frequency experiment," Journal of Geophysical Research, vol. 104, no. 10, pp. 13-13, 1999.

[32] S. Leinss, H. Löwe, M. Proksch, J. Lemmetyinen, A. Wiesmann, and I. Hajnsek, "Anisotropy of seasonal snow measured by polarimetric phase differences in radar time series," The Cryosphere, vol. 10, pp. 1771-1797, 2016.

[33] R. Touzi and A. Lopes, "Coherence Estimation for SAR Imagery," IEEE Transactions of Geoscience and Remote Sensing, vol. 37, no. 1, pp. 135149, 1999.

[34] D. T. Sandwell and E. J. Price, "Phase gradient approach to stacking interferograms ," Journal of Geophysical Research, vol. 103, pp. $30183-$ 30204,1998

[35] N. B. Karlsson, D. Dahl-Jensen, S. P. Gogineni, and J. D. Paden, "Tracing the depth of the Holocene ice in North Greenland from radioecho sounding data," Annals of Glaciology, vol. 54, no. 64, pp. 44-50, 2013.

[36] W. Chu, D. M. Schroeder, H. S. Seroussi, T. T. Creyts, and R. E. Bell, "Complex basal thermal transition near the onset of Petermann Glacier, Greenland," Journal of Geophysical Research: Earth Surface, vol. 123, p. 2017JF004561, 2018.

[37] D. Dahl-Jensen et al., "Eemian interglacial reconstructed from a Greenland folded ice core," Nature, vol. 493, no. 7433, pp. 489-494, 2013.

[38] C. Martín, G. H. Gudmundsson, H. D. Pritchard, and O. Gagliardini, "On the effects of anisotropic rheology on ice flow, internal structure, and the age-depth relationship at ice divides," Journal of Geophysical Research: Earth Surface, vol. 114, no. 4, pp. 1-18, 2009. 